\title{
Just say no? Public attitudes about supportive and punitive policies to combat the opioid epidemic
}

\author{
Steven M. Sylvester ${ }^{1}$ (D), Simon F. Haeder ${ }^{2 *}$ (iD) and Timothy Callaghan ${ }^{3}$ \\ ${ }^{1}$ Assistant Professor, Department of History and Political Science, Utah Valley University, USA, ${ }^{2}$ Assistant \\ Professor of Public Policy, School of Public Policy, The Pennsylvania State University, USA and ${ }^{3}$ Assistant \\ Professor, Department of Health Policy and Management, Texas A\&M University, USA \\ ${ }^{*}$ Corresponding author. E-mail: sfh5482@psu.edu
}

(Received 3 September 2020; revised 28 June 2021; accepted 29 June 2021; first published online 09 February 2022)

\begin{abstract}
Using an original demographically representative survey, we estimate the determinants of public support for a set of supportive and punitive policies to combat the opioid epidemic among a sample of 2,131 Americans. Our findings indicate that individuals who attribute blame for the epidemic to the personal choices of individuals, conservatives and those high in racial resentment are consistently more likely to support punitive policies to combat the opioid epidemic and less likely to favour policies to support individuals with substance use disorders. Individuals who have a personal connection to someone struggling with opioid use disorder favour policies to support such individuals but have nuanced attitudes towards punitive policies. Importantly, we find overwhelming support for all supportive policies except supervised injection sites, while roughly $50 \%$ of our sample supported the set of punitive policy choices. Our research represents a significant step forward toward understanding public opinion about the opioid epidemic and policies to combat it.
\end{abstract}

Key words: attribution theory; opioids; public opinion; substance use disorders

The opioid epidemic in the United States (US) has surged in recent years, with 67,367 Americans dying from an opioid overdose in just 2018 (Center for Disease Control and Prevention 2019). Since 1999, it has claimed over 750,000 deaths and wreaked havoc in families and communities across the US (Center for Disease Control and Prevention 2019). As the opioid epidemic has worsened in the US in recent years, federal and state governments have sought policy solutions to combat the epidemic. Most notably, former President Trump declared the opioid epidemic a public health emergency, unlocking federal funds for states and local governments to reduce the supply and demand of illicit drugs and thus reduce

(C) The Author(s), 2022. Published by Cambridge University Press. This is an Open Access article, distributed under the terms of the Creative Commons Attribution licence (http://creativecommons.org/licenses/by/4.0/), which permits unrestricted re-use, distribution and reproduction, provided the original article is properly cited. 
inappropriate opioid use in the US (White House 2018). ${ }^{1}$ More recently, efforts to fight the opioid epidemic have confronted a new set of challenges as the coronavirus pandemic has increased stressors for many Americans while negatively impacting access to substance use disorder (SUD) treatment, further increasing opioid mortality rates (American Medical Association 2020).

One common narrative surrounding the opioid epidemic has emphasised those experiencing SUD and blaming individuals for their circumstances and the epidemic's growth. This is, of course, in line with past narratives surrounding SUDs, and Nancy Reagan's famous argument that users could have just said no to drugs vividly comes to mind (Quenqua 2016). Similarly, President Trump has argued that "If they [users] don't start, they won't have a problem" (White House 2017). This narrative has also been used by street-level bureaucrats within local communities like the tough law-and-order sheriff in Butler County, $\mathrm{OH}$, who refused to let his deputies carry Narcan (Wootson 2017).

As the scope of the opioid epidemic has grown in recent years, other narratives explaining opioid use disorder (OUD) have gained prominence as well. For example, one emerging narrative has been to place blame at the feet of health professionals and, in particular, pharmaceutical companies such as Purdue Pharma, the maker of the narcotic OxyContin. The prescription drug has been prominently featured in media reports detailing how the company sought to expand the use of prescription opioids by incentivizing sales staff to push OxyContin to "pill mills" (Lopez 2017; Taddonio 2019). More recently, this narrative has been amplified by dozens of state attorneys general seeking restitution from opioid manufacturers and their owners for the damage the crisis has done to their states (Lopez 2019).

Given these conflicting narratives alternatively blaming individuals who use substances themselves or the healthcare industry, it is not surprising that while most Americans see OUD as a severe health and social issue facing the US, there is less consensus on the policies that are needed to combat the epidemic (see Kaiser Family Foundation 2016). We posit that the disparity between the widespread acceptance of OUD as a problem and variation in support for strategies to fight the opioid epidemic is partially due to disagreements over the root causes of the opioid crisis among the public and policymakers. We thus argue that the attribution of blame for the epidemic has important, albeit understudied, policy consequences. Most importantly, by influencing support for and opposition to policies designed to address the opioid epidemic, it may make policymakers hesitant to push for certain types of policy solutions like supervised injection facilities (SIFS) even though they have been found to be effective (Beletsky et al. 2008). The dialogue about the causes of the opioid epidemic thus provides an intriguing context for the study of attribution and its influence on policy attitudes and, ultimately, policymaking and implementation.

\footnotetext{
${ }^{1}$ President Trump's plan included reducing the demand for over-the-counter prescription drugs by raising public awareness about the dangers of prescription drugs. The initiative also ensured that first responders were supplied with naloxone and provided federal funding to state and local jurisdictions to incentivise a nationwide overdose tracking system. For more information, see https://www.whitehouse.gov/briefingsstatements/president-donald-j-trumps-initiative-stop-opioid-abuse-reduce-drug-supply-demand/.
} 
To explore the role of blame attribution in the opioid epidemic, our study utilises attribution theory as a theoretical framework to help explain policy preferences about the opioid epidemic. We posit that the way individuals view the causes of the opioid epidemic will influence their preferences towards policies designed to combat it. Specifically, we anticipate that to whom individuals' attribute the causes of the epidemic, that is, either to personal choices (factors controlled by the individual) or situational factors (factors outside the control of the individual), will exert a substantial effect on what type of policies they support to mitigate the epidemic. Simultaneously, other factors are also likely to influence attitudes towards policies to combat the opioid epidemic. We argue that attitudes may be influenced by a personal connection to someone coping with SUD or having personal experience with SUD. The first-hand knowledge these individuals gain about the struggles those suffering from SUD face daily could inform their preferences for policy. Furthermore, we hypothesise that ideology and racial resentment will exert a substantive effect on policy attitudes.

In the discussion below, we begin by briefly describing attribution theory and its potential role in explaining attitudes toward policies to combat the opioid epidemic. We then lay out expectations for determinants of policy preferences towards solutions for the opioid epidemic, focusing on attributions of blame, personal connections, political ideology and racial resentment. Our analysis suggests that attributions of blame influence policy preferences related to combatting the opioid epidemic. We show that individuals who attribute OUD to personal choices are consistently more likely to support policies designed to punish individuals and are less likely to favour policies designed to aid individuals overcoming SUD/OUD. We also find consistent effects for the impact of ideology and racial resentment on attitudes. However, while the findings for personal connection and experience align with our expectations for supportive policies, they are more nuanced for punitive ones. We conclude by discussing the implication of our findings for health policy and combatting the epidemic by noting directions for future research.

\section{Factors influencing attitudes toward opioid policies}

As described above, we rely on attribution theory as the theoretical framework that informs our expectations about public opinion related to the opioid epidemic. In his seminal work on attributions, Fritz Heider $(1944,1958)$ examined how individuals assess the causes and consequences of human behaviour. These inferences arise from interactions with the environment throughout the lifetime of an individual. For example, an individual may encounter a homeless person and attribute the cause of their homelessness to the personal choices of that specific individual, or instead to situational factors like the economy, employment opportunities, systemic biases or family dynamics. ${ }^{2}$ The inferences we make about SUD can be similar to the inferences an individual could make about homelessness. Numerous studies using attribution theory have confirmed and significantly extended Heider's original theory to various policy fields, including obesity (Cozzarelli et al. 2002; Haider-Markel

\footnotetext{
${ }^{2}$ These specific factors are only used as examples of situational factors that could influence SUD and were not explicitly tested in our analysis.
} 
and Joslyn 2018), gay rights (Haider-Markel and Joslyn 2008), gun violence (Joslyn and Haider-Markel 2017), poverty (Zucker and Weiner 1993) and smartphone addiction (Jeong et al. 2018).

\section{Attributions of blame and opioid addiction}

We theorise that individuals make inferences toward the underlying causes of SUD/ OUD, which affect how individuals attribute blame for an individual becoming addicted to opioids. Specifically, individuals' explanations can be grouped into two categories. Internal attributions focus on the individual (i.e. personal choices), while external attributions focus more on the environment (i.e. situational factors outside the control of the individual). A critical third dimension of how blame is assigned is the notion of controllability. Weiner's $(1995,2006)$ controllability dimension proposed that individuals are less willing to support (and more willing to punish) when they perceive that the cause of a problem is due to an individual's own decisions and less willing to punish when they perceive the cause of the problem is beyond the control of the individual. The emphasis on controllability is beneficial when trying to explain public attitudes towards stigmatised topics. Drug addiction falls into this category as it is one of the most stigmatised conditions in society (Corrigan et al. 2009; Barry et al. 2014).

Individuals with SUD are often perceived to be personally blamed for their addiction, with many supporting the narrative that individuals suffering from SUD lack the self-discipline to properly use drugs - particularly prescription opioids - without becoming a person with SUD (Kennedy-Hendricks et al. 2017). Existing research has examined the influence of attributions on the way individuals view policies to combat the opioid epidemic (Barry et al. 2016; Kennedy-Hendricks et al. 2017; Goodyear et al. 2018; Haeder et al. 2021). However, the literature has yet to fully incorporate psychological dispositions, social variables and political variables into studies of attribution related to the epidemic and consider their impact on punitive and supportive policies. We posit that accounting for these specific factors, the way individuals view the causes of the opioid epidemic, as either internally or externally driven, will shape how they view policies to battle the opioid epidemic. That is, those individuals who feel addiction to opioids is a personal choice will be less likely to support policies designed to support those suffering from OUD, and they will prefer policies that are designed to punish (Hypothesis \#1).

\section{Personal connection}

While the notions of attribution and controllability are important to consider when evaluating public attitudes toward opioid policies, just as important may be a personal connection to an individual who suffers from OUD. The influence of personal connections on individual policy preferences is an important predictor in other areas of health policy, most notably with policy preferences towards Medicaid. For example, Grogan and Park $(2017,2018)$ found that individuals with any connection to the Medicaid program were more likely to view the program as an important federal policy than those who had no connection to the program. 
Some research suggests that being familiar with SUD/OUD (i.e. having contact with someone dealing with OUD/SUD) can play an important role in determining attitudes (see Addison and Thorpe 2004; Corrigan et al. 2009; Sattler et al. 2017). However, research on whether having a personal connection to someone with OUD/SUD influences attitudes and preferences has produced mixed results. For example, Barry et al. (2016) found that those with a personal experience with the opioid crisis, through either themselves, a family member or a close friend, were more likely to gravitate towards individual-orientated causes of OUD, which includes self-discipline. Other research, however, has found no significant difference between the attitudes of those with a personal connection to OUD and those without a personal connection to OUD (Kennedy-Hendricks et al. 2017; Haeder et al. 2021). Because of the mixed findings in the extant literature, it is important to investigate how personal connections influence individual policy preferences about potential solutions to the opioid epidemic. We expect that individuals who connect to an individual suffering from OUD will be more likely to support policies designed to help overcome the disorder and will be less likely to support policies meant to punish individuals suffering from OUD (Hypothesis \#2a). We expect that the same effect to be present for individuals who personally suffer from OUD (Hypothesis \#2b).

\section{Political ideology}

Highlighting the importance of political measures on individual attitudes towards policy, a growing body of research shows that an individual's perception of blame can influence policy preferences. For example, evidence suggests that liberals are more likely to perceive the structural causes of poverty, which are beyond an individual's control, as important (Furnham 1982; Pandey et al. 1982; S. Williams 1984; Bullock 1999; Skitka 1999; Cozzarelli et al. 2002; Bullock et al. 2003; Haeder et al. 2020). That is, liberals attribute the root causes of poverty to situational factors like discriminatory governmental policies, a lack of educational opportunity and outright racism. This leads liberals to be more likely to support policies meant to aid individuals out of poverty through social programs like Medicaid and unemployment benefits.

On the other hand, conservatives are more likely to point to individuals as being responsible for their circumstances. In the case of poverty, some conservatives believe that economic circumstances are at least partially attributable to individuals themselves and feel individuals can and should pull themselves up out of poverty through hard work; a failure to do so can be ascribed to laziness and poor personal choices like SUD (Pandey et al. 1982; Griffin and Oheneba-Sakyi 1993; Bullock 1999; Bullock et al. 2003; Hopkins 2009). Conservatives are hence much less likely to support extending unemployment benefits and have actively worked to make it harder to access many social safety net programs designed to assist individuals out of poverty. These causal attributions and subsequent policy preferences often extend beyond poverty and welfare policy (Haider-Markel and Joslyn 2008). We expect conservatives to be more likely to support policies that criminalise the use of opioids. At the same time, liberals will be more likely to support policies designed to aid individuals overcoming OUD (Hypothesis \#3). 


\section{Racial resentment}

Finally, the last key factor to consider, especially when studying stigmatised issues like drug use and addiction, is racial resentment. Research has demonstrated that for many people of colour, and Black Americans in particular, their direct interactions with the government comes in the form of punishment (Weaver 2007). Elected officials have regularly used race as a mechanism to appeal to Whites, most notably with the presidential campaigns of Nixon and recently with former President Trump, tapping into the rising rates of racial resentment among Whites in recent years (Baker et al. 2019; Jardina 2019).

However, the extent to which racial attitudes shape White Americans' attitudes toward public policy depends on the policy domain. One area where race has been a primary focus of rhetoric and discourse is criminal justice policy. For example, Enns and Ramirez (2018) found that support for privatising prisons and immigration detention facilities was influenced by racial resentment. Similarly, Morris and LeCount (2020) found that racial resentment predicts support for increased spending on law enforcement. Scholars have long argued that criminal justice changes in the US have been used as a mechanism to reassert control over Black Americans (Bobo and Johnson 2004; Weaver 2007).

Racial attitudes have also been found to influence policy preferences within social welfare policy. Research has shown that many Whites believe redistributive programs primarily benefit people of colour, although most redistributive programs primarily benefit Whites (Tesler 2016; Callaghan and Olson 2017; Wetts and Willer 2018). This includes assistance for low-income seniors, unemployment insurance and parental leave benefits (Harell et al. 2016). Some scholars have even found that racial resentment influences attitudes towards projects that could benefit their state (Krimmel and Rader 2017). Finally, recent public health policy issues, particularly the Affordable Care Act, are significantly affected by racial resentment (Tesler 2012; Haeder 2020).

Much like criminal justice policy, the connection between punitive policies and racial resentment has been used to explain individual attitudes towards drug use. Racial bias has long been associated with drug policy, most notably, with how crack cocaine use was punished more severely than powdered cocaine use, disproportionately affecting Blacks (Bobo and Johnson 2004). Other research has found, for example, that white respondents who hold prejudicial attitudes toward African Americans and Latinos are less likely to say that too little money is spent on drug rehabilitation (Nielsen Bonn, and Wilson (2010). Based on this body of literature demonstrating the impact of racial resentment on policy attitudes and drug use, we expect individual attitudes toward policies designed to combat the opioid epidemic to be influenced by racial resentment, much like attitudes towards criminal justice, social welfare, health and other drug policy. Specifically, we expect that individuals who hold higher levels of racial resentment will be more likely to support policies meant to punish individuals suffering from OUD and less likely to support policies meant to assist individuals to overcome OUD (Hypothesis \#4). 


\section{Data and methods}

To test our hypotheses, we fielded a demographically representative online survey in December 2018 administered through Qualtrics (Sylvester et al. 2021). Qualtrics relies on large, online opt-in panels to recruit respondents to take surveys, an approach suitable for our analyses here. ${ }^{3}$ We received 2,131 responses, including an oversample of 754 LGBTQ individuals (whose data was collected for a separate project also included in the survey). ${ }^{4}$ We weighted the data to reflect national population benchmarks on gender, race, income and education, which we drew from the US. Census 2017 Current Population Survey. ${ }^{5}$ Although the unweighted survey data are close to Census benchmarks, Table A1 in the supplemental material shows that the weights improve representativeness. ${ }^{6}$

\section{Dependent variables}

Our primary interest in this study is to examine the correlates of attitudes towards policies designed to combat the opioid epidemic. For our dependent variables, we asked respondents their opinions about various policies associated with the opioid epidemic. We categorised these policies into two themes: (1) policies designed to aid individuals to overcome OUD and (2) policies designed to punish individuals suffering from OUD. Respondents were asked to state whether they favoured or opposed the various policies on a five-point scale ranging from "strongly favor" to "strongly oppose." The full wording of the survey questions used in our analysis can be found in the supplemental materials.

\section{Explanatory measures}

In order to gauge whom Americans hold responsible for the current opioid epidemic, we asked respondents to think about why people become addicted to prescription opioids. In order to measure attributions of blame (Personal Choice), respondents were asked to choose between two possible answers: whether OUD is caused by the personal choices of the users themselves or due to some other factor. ${ }^{7}$

\footnotetext{
${ }^{3}$ Although our analysis relies on an opt-in panel to recruit respondents, the Pew Research Centre suggests that high-quality non-probability samples like ours produce similar results to probability-based surveys. Additionally, considerable research in the social sciences demonstrates that opt-in panels like ours are appropriate when modelling relationships between variables (Coppock and McClellan 2019; Levay et al. 2016; Baker et al. 2013; Pew Research Center 2016).

${ }^{4}$ The LGBT portion of the sample was down-weighted to reflect the proportion of the US population identified as LGBT.

${ }^{5}$ Qualtrics respondents were identified from the survey panels of Research Now and Lucid. Supporting the quality of our sample, work by Coppock and McClellan (2019) finds that data from Lucid - which Qualtrics used to select our participants, are comparative population benchmarks, much better than most convenience samples, and "suitable for evaluating many social scientific theories."

${ }^{6}$ The institutional review boards at Texas A\&M University, West Virginia University, The Pennsylvania State University, and Utah Valley University approved the study.

${ }^{7}$ If respondents answered "some other factor" for why some people become addicted to opioids, they were provided various options to choose from like the health industry, drug dealers, the federal government, genetic factors the person was born with or other factors.
} 
Our analysis also included a measure to gauge how having a personal connection to the opioid epidemic influences policy preferences. To measure this, we asked respondents if they personally knew anyone who has ever been addicted to prescription opioids or heroin to create our measure of Personal Connection. Respondents were coded with a 1 if they personally knew someone who was addicted to opioids and 0 if they did not personally know anyone. ${ }^{8}$ Simultaneously, we also accounted for whether the respondent has suffered from OUD.

Recall that we also are interested in how political ideology and racial resentment influence policy preferences toward opioids. Our measure of Ideology was created by using the standard approach of asking respondents to describe their political views on a seven-point scale ranging from "extremely liberal" to "extremely conservative." Racial Resentment was measured using a four-question scale developed in previous work by Kinder and Sanders (1996) meant to tap into racial predispositions of individuals. ${ }^{9}$

Finally, we also control for a variety of other demographics in our models. These include gender (a dichotomous measure with female coded as 1); race (using separate indicators for whether participants are Black or Hispanic); age and its squared term; educational attainment (we include indicator variables for high school degree, high school plus college and bachelor degree or higher with less than high school as the reference category); household income (on a seven-point scale included as indicators with the lowest income category as reference); indicators for rural and urban residence (with suburban as reference); insurance coverage type (indicators for employer-sponsored coverage and Medicare); political knowledge (on a seven-point scale) and religiosity (a measure to assess respondents level of church attendance on a five-point scale).

\section{Results}

\section{The effect of causal attributions on policy preferences}

Policies designed to support treatment efforts for opioid addiction

As described above, we asked respondents about their preferences for several policies that were either supportive of individuals suffering from OUD or more punitive policies. We selected policy options based on those policies currently discussed among policymakers as potential solutions to mitigate the opioid epidemic. Policy options designed to aid those addicted to opioids include implementing Good Samaritan protections (Table 1: Model 1), allowing for SIFS (Table 1: Model 2), ${ }^{10}$ expanding access to drug testing kits (Table 1: Model 3), ${ }^{11}$ creating

\footnotetext{
${ }^{8} \mathrm{We}$ conducted a robustness check to see if the results would change if we included a variable that examined the respondents' connection to the opioid crisis was within their immediate family or not. None of the results were substantively different, so we opted for the more generalisable measure.

${ }^{9}$ Recently there has been some criticism of the Kinder and Sanders (1996) racial resentment scale, most notably by Kam \& Burge (2018) and DeSante and Smith (2020). Both argue that the Kinder \& Sanders (1996) racial resentment scale measures aspects of conservative ideology. We acknowledge these concerns in our limitation and note that while our racial resentment scale is correlated to conservatism $(\mathrm{R}=0.3875)$, our variance inflation factor indicated no multicollinearity problems in our analysis.

${ }^{10}$ SIFS are locations monitored by a licensed health professional to provide individuals a safe and clean environment to reduce drug overdoses.

${ }^{11}$ Drug kits refer to kits that can be purchased, which can test opioids and heroin for fentanyl, a synthetic opioid that is $50-100$ times more potent than heroin (Harris 2017).
} 
Table 1a. Estimates for supportive policies, all respondents

\begin{tabular}{|c|c|c|c|c|c|c|c|}
\hline & (1) & (2) & (3) & (4) & (5) & (6) & (7) \\
\hline Variables & Good Samaritan & Safe sites & Test Kits & Diversion & Medicaid & Insurance & Marijuana \\
\hline Personal choice & $\begin{array}{c}-0.425^{\star \star \star} \\
(0.000)\end{array}$ & $\begin{array}{c}0.175 \\
(0.120)\end{array}$ & $\begin{array}{c}-0.148 \\
(0.192)\end{array}$ & $\begin{array}{c}-0.331^{\star *} \\
(0.004)\end{array}$ & $\begin{array}{c}-0.660^{\star \star \star} \\
(0.000)\end{array}$ & $\begin{array}{c}-0.642^{\star \star \star} \\
(0.000)\end{array}$ & $\begin{array}{c}-0.612^{\star * *} \\
(0.000)\end{array}$ \\
\hline Opioid use disorder & $\begin{array}{l}1.081^{\star *} \\
(0.005)\end{array}$ & $\begin{array}{c}0.711^{*} \\
(0.050)\end{array}$ & $\begin{array}{c}0.754^{\star} \\
(0.027)\end{array}$ & $\begin{array}{l}1.384^{\star \star \star} \\
(0.000)\end{array}$ & $\begin{array}{l}1.313^{\star \star \star} \\
(0.000)\end{array}$ & $\begin{array}{l}1.467^{\star \star \star} \\
(0.000)\end{array}$ & $\begin{array}{l}0.973^{\star \star \star} \\
(0.001)\end{array}$ \\
\hline Personal connection & $\begin{array}{l}0.424^{\star \star \star} \\
(0.001)\end{array}$ & $\begin{array}{c}0.053 \\
(0.649)\end{array}$ & $\begin{array}{l}0.354^{\star \star} \\
(0.003)\end{array}$ & $\begin{array}{l}0.562^{\star \star \star} \\
(0.000)\end{array}$ & $\begin{array}{l}0.417^{\star \star \star} \\
(0.000)\end{array}$ & $\begin{array}{l}0.323^{\star \star} \\
(0.006)\end{array}$ & $\begin{array}{l}0.797^{\star \star \star} \\
(0.000)\end{array}$ \\
\hline Ideology & $\begin{array}{c}-0.209^{\star \star \star} \\
(0.000)\end{array}$ & $\begin{array}{c}-0.335^{\star \star \star} \\
(0.000)\end{array}$ & $\begin{array}{c}-0.163^{\star \star \star} \\
(0.000)\end{array}$ & $\begin{array}{c}-0.155^{\star \star \star} \\
(0.000)\end{array}$ & $\begin{array}{c}-0.268^{\star \star \star} \\
(0.000)\end{array}$ & $\begin{array}{c}-0.259^{\star \star \star} \\
(0.000)\end{array}$ & $\begin{array}{c}-0.222^{\star \star \star} \\
(0.000)\end{array}$ \\
\hline Religiosity & $\begin{array}{r}-0.107^{\star} \\
(0.018)\end{array}$ & $\begin{array}{c}-0.082 \\
(0.052)\end{array}$ & $\begin{array}{l}-0.151^{\star \star \star} \\
(0.000)\end{array}$ & $\begin{array}{r}-0.096^{*} \\
(0.022)\end{array}$ & $\begin{array}{c}-0.009 \\
(0.839)\end{array}$ & $\begin{array}{c}-0.003 \\
(0.945)\end{array}$ & $\begin{array}{c}0.112^{\star} \\
(0.013)\end{array}$ \\
\hline Female & $\begin{array}{c}-0.093 \\
(0.461)\end{array}$ & $\begin{array}{c}-0.393^{\star \star} \\
(0.002)\end{array}$ & $\begin{array}{c}-0.088 \\
(0.472)\end{array}$ & $\begin{array}{c}-0.055 \\
(0.661)\end{array}$ & $\begin{array}{c}-0.117 \\
(0.332)\end{array}$ & $\begin{array}{c}0.265^{\star} \\
(0.032)\end{array}$ & $\begin{array}{c}-0.291^{\star} \\
(0.031)\end{array}$ \\
\hline Black & $\begin{array}{c}-0.149 \\
(0.424)\end{array}$ & $\begin{array}{c}-0.152 \\
(0.371)\end{array}$ & $\begin{array}{c}0.141 \\
(0.435)\end{array}$ & $\begin{array}{c}-0.028 \\
(0.875)\end{array}$ & $\begin{array}{c}-0.208 \\
(0.232)\end{array}$ & $\begin{array}{c}-0.101 \\
(0.551)\end{array}$ & $\begin{array}{c}-0.152 \\
(0.395)\end{array}$ \\
\hline Hispanic & $\begin{array}{c}0.068 \\
(0.683)\end{array}$ & $\begin{array}{c}0.372^{\star} \\
(0.033)\end{array}$ & $\begin{array}{c}0.427^{\star} \\
(0.019)\end{array}$ & $\begin{array}{c}0.332 \\
(0.085)\end{array}$ & $\begin{array}{c}0.223 \\
(0.204)\end{array}$ & $\begin{array}{c}0.304 \\
(0.095)\end{array}$ & $\begin{array}{c}0.300 \\
(0.099)\end{array}$ \\
\hline Rural & $\begin{array}{c}0.178 \\
(0.230)\end{array}$ & $\begin{array}{c}-0.128 \\
(0.356)\end{array}$ & $\begin{array}{c}0.040 \\
(0.780)\end{array}$ & $\begin{array}{c}-0.055 \\
(0.702)\end{array}$ & $\begin{array}{c}-0.210 \\
(0.122)\end{array}$ & $\begin{array}{c}0.001 \\
(0.994)\end{array}$ & $\begin{array}{c}0.100 \\
(0.507)\end{array}$ \\
\hline Urban & $\begin{array}{c}0.135 \\
(0.344)\end{array}$ & $\begin{array}{c}0.096 \\
(0.469)\end{array}$ & $\begin{array}{c}0.181 \\
(0.187)\end{array}$ & $\begin{array}{c}0.053 \\
(0.709)\end{array}$ & $\begin{array}{c}0.184 \\
(0.196)\end{array}$ & $\begin{array}{c}0.257 \\
(0.051)\end{array}$ & $\begin{array}{c}0.016 \\
(0.915)\end{array}$ \\
\hline Age & $\begin{array}{l}0.053^{\star \star} \\
(0.004)\end{array}$ & $\begin{array}{c}-0.009 \\
(0.591)\end{array}$ & $\begin{array}{l}0.051^{\star \star} \\
(0.003)\end{array}$ & $\begin{array}{l}0.056^{\star \star} \\
(0.002)\end{array}$ & $\begin{array}{c}0.040^{\star} \\
(0.019)\end{array}$ & $\begin{array}{l}0.078^{\star \star \star} \\
(0.000)\end{array}$ & $\begin{array}{l}0.080^{\star \star \star} \\
(0.000)\end{array}$ \\
\hline Age squared & $\begin{array}{r}-0.000^{\star} \\
(0.021)\end{array}$ & $\begin{array}{c}0.000 \\
(0.602)\end{array}$ & $\begin{array}{c}-0.001^{\star \star} \\
(0.004)\end{array}$ & $\begin{array}{c}-0.000^{\star *} \\
(0.006)\end{array}$ & $\begin{array}{c}-0.000^{\star *} \\
(0.006)\end{array}$ & $\begin{array}{c}-0.001^{\star \star *} \\
(0.001)\end{array}$ & $\begin{array}{c}-0.001^{\star \star \star} \\
(0.000)\end{array}$ \\
\hline Political knowledge & $\begin{array}{c}0.082^{\star} \\
(0.049)\end{array}$ & $\begin{array}{c}-0.175^{\star \star \star} \\
(0.000)\end{array}$ & $\begin{array}{c}0.008 \\
(0.848)\end{array}$ & $\begin{array}{l}0.126^{\star \star} \\
(0.006)\end{array}$ & $\begin{array}{c}0.062 \\
(0.184)\end{array}$ & $\begin{array}{c}0.059 \\
(0.176)\end{array}$ & $\begin{array}{c}0.097^{\star} \\
(0.030)\end{array}$ \\
\hline Employer-sponsored & $\begin{array}{c}0.078 \\
(0.580)\end{array}$ & $\begin{array}{c}-0.052 \\
(0.715)\end{array}$ & $\begin{array}{c}-0.219 \\
(0.122)\end{array}$ & $\begin{array}{c}-0.066 \\
(0.644)\end{array}$ & $\begin{array}{c}0.146 \\
(0.320)\end{array}$ & $\begin{array}{c}0.189 \\
(0.174)\end{array}$ & $\begin{array}{c}-0.079 \\
(0.576)\end{array}$ \\
\hline Medicare & $\begin{array}{c}-0.061 \\
(0.728)\end{array}$ & $\begin{array}{c}-0.049 \\
(0.758)\end{array}$ & $\begin{array}{c}-0.004 \\
(0.982)\end{array}$ & $\begin{array}{c}-0.165 \\
(0.300)\end{array}$ & $\begin{array}{c}-0.108 \\
(0.536)\end{array}$ & $\begin{array}{c}-0.114 \\
(0.528)\end{array}$ & $\begin{array}{c}-0.100 \\
(0.571)\end{array}$ \\
\hline Education & Yes & Yes & Yes & Yes & Yes & Yes & Yes \\
\hline Income & Yes & Yes & Yes & Yes & Yes & Yes & Yes \\
\hline McKelvey and Zavoina $\mathrm{R}^{2}$ & 0.125 & 0.173 & 0.088 & 0.088 & 0.151 & 0.150 & 0.173 \\
\hline Observations & 2,007 & 2,009 & 1,994 & 1,997 & 2,003 & 1,995 & 1,996 \\
\hline
\end{tabular}

Robust $\mathrm{p}$-values in parentheses ${ }^{\star \star *} \mathrm{p}<0.001,{ }^{\star \star} \mathrm{p}<0.01,{ }^{*} \mathrm{p}<0.05$. 
diversion programs like drug courts (Table 1: Model 4), allowing Medicaid to pay for rehabilitation services (Table 1: Model 5), mandating private insurance to pay for SUD treatment (Table 1: Model 6) and legalising marijuana (Table 1: Model 7). ${ }^{12}$

To assess support or opposition for the various policy options, we asked respondents whether they strongly favour, somewhat favour, somewhat oppose or strongly oppose adopting the various policies. We also offered respondents a neutral option. Due to the ordinal nature of data, we relied on standard ordered logit models, also known as proportional odds models, for our analyses presented here (R. Williams 2016). ${ }^{13}$ Interpretation of ordered logit models is not straightforward. To facilitate interpretation, we rely on average marginal effects (AMEs) as well as predicted probabilities (Cameron and Trivedi 2010; Long and Freese 2014). For both, we utilise graphical displays. Finally, we also estimated standard ordinary least squares (OLS) regression models as a robustness check of our findings. ${ }^{14}$

Results for policies designed to aid those suffering from OUD are shown in Tables $1 \mathrm{a}$ and $1 \mathrm{~b}$, with the former displaying the results for all respondents and the latter displaying the results for non-Hispanic White respondents only. ${ }^{15}$ Across the two tables, we found that coefficient signs and significance were in line with our expectations. Coefficients for Personal Choice (with two exceptions) and Ideology were negatively signed and significant, indicating that those who saw addiction as a personal choice and conservatives were more strongly opposed to policies assisting those struggling with OUD. The opposite holds for those who have suffered from OUD (Addiction) and for those with Personal Connections to someone with an OUD (with one exception).

AMEs offer additional help in interpreting the results, particularly for policyrelevant research, because they illustrate how substantively and statistically significant effects are (Cameron and Trivedi 2010). To provide context, we present the average predictions for all models from Table $1 \mathrm{a}$ and $1 \mathrm{~b}$ in Figure 1 . The figure displays the distribution of respondents along with the five-point scales of the dependent variables. Focusing on the results for all respondents first, we note that for six of the seven policies, probabilities of falling in the two favourable categories (somewhat and strong favour) exceed 0.5 , and in four of the seven cases, 0.6. Notably, in six of seven cases, the oppositional categories do not exceed 0.2. The policy that stands out among the seven choices is the creation of SIFS. Here, support and opposition both have probabilities of roughly 0.4 , with the remainder falling in the neutral category. When we compare the average predictions for all respondents to those for non-Hispanic Whites only, the results indicate general congruence, with some differences in support for SIFS and drug test kits (more opposition among nonHispanic Whites). However, it is perhaps unsurprising that the public is more likely to oppose implementing SIFS. First, there are no SIFS located in the US to serve as a

\footnotetext{
${ }^{12} \mathrm{We}$ split the table into $1 \mathrm{a}$ for all respondents and $1 \mathrm{~b}$ for non-Hispanic Whites to facilitate our display.

${ }^{13} \mathrm{We}$ relied on gologit2 to assess the parallel regression assumptions (R. Williams 2016).

${ }^{14}$ These OLS model robustness checks are omitted here but available on request.

${ }^{15}$ While research has shown that Blacks can hold levels of racial resentment toward their own in-group (Kam \& Burge, 2018), adding Blacks to the analysis did not significantly change the results. All respondents were given the racial resentment scale on the survey. However, we only included analysis of racial resentment for non-Hispanic whites within the article to remain consistent with the existing literature using racial resentment to study policy attitudes.
} 
Table 1b. Estimates for supportive policies, White non-Hispanics

\begin{tabular}{|c|c|c|c|c|c|c|c|}
\hline & $(1)$ & $(2)$ & (3) & (4) & (5) & (6) & (7) \\
\hline Variables & Good Samaritan & Safe Sites & Test Kits & Diversion & Medicaid & Insurance & Marijuana \\
\hline Personal choice & $\begin{array}{c}-0.259 \\
(0.093)\end{array}$ & $\begin{array}{c}0.257 \\
(0.075)\end{array}$ & $\begin{array}{c}-0.137 \\
(0.340)\end{array}$ & $\begin{array}{c}-0.206 \\
(0.160)\end{array}$ & $\begin{array}{l}-0.675^{\star \star \star} \\
(0.000)\end{array}$ & $\begin{array}{l}-0.560^{\star \star \star} \\
(0.000)\end{array}$ & $\begin{array}{c}-0.612^{\star \star \star} \\
(0.000)\end{array}$ \\
\hline Opioid use disorder & $\begin{array}{l}1.270^{\star \star} \\
(0.006)\end{array}$ & $\begin{array}{c}0.593 \\
(0.220)\end{array}$ & $\begin{array}{c}1.068^{\star} \\
(0.016)\end{array}$ & $\begin{array}{l}2.028^{\star \star \star} \\
(0.000)\end{array}$ & $\begin{array}{l}1.640^{\star \star \star} \\
(0.000)\end{array}$ & $\begin{array}{l}1.726^{\star \star \star} \\
(0.000)\end{array}$ & $\begin{array}{l}1.389^{\star \star \star} \\
(0.000)\end{array}$ \\
\hline Personal connection & $\begin{array}{l}0.535^{\star \star \star} \\
(0.000)\end{array}$ & $\begin{array}{c}0.017 \\
(0.906)\end{array}$ & $\begin{array}{c}0.353^{\star} \\
(0.015)\end{array}$ & $\begin{array}{l}0.697^{\star \star \star} \\
(0.000)\end{array}$ & $\begin{array}{l}0.526^{\star \star \star} \\
(0.000)\end{array}$ & $\begin{array}{l}0.469^{\star \star} \\
(0.001)\end{array}$ & $\begin{array}{l}0.919^{\star \star \star \star} \\
(0.000)\end{array}$ \\
\hline Ideology & $\begin{array}{c}-0.152^{\star \star} \\
(0.003)\end{array}$ & $\begin{array}{c}-0.227^{\star \star \star} \\
(0.000)\end{array}$ & $\begin{array}{c}-0.120^{\star *} \\
(0.009)\end{array}$ & $\begin{array}{c}-0.143^{\star *} \\
(0.004)\end{array}$ & $\begin{array}{l}-0.229^{\star \star \star} \\
(0.000)\end{array}$ & $\begin{array}{l}-0.241^{\star \star *} \\
(0.000)\end{array}$ & $\begin{array}{c}-0.196^{\star \star \star} \\
(0.000)\end{array}$ \\
\hline Racial resentment & $\begin{array}{c}-1.658^{\star \star \star} \\
(0.000)\end{array}$ & $\begin{array}{c}-2.952^{\star * \star} \\
(0.000)\end{array}$ & $\begin{array}{c}-1.272^{\star \star \star} \\
(0.000)\end{array}$ & $\begin{array}{c}-1.183^{\star *} \\
(0.002)\end{array}$ & $\begin{array}{c}-1.875^{\star \star \star} \\
(0.000)\end{array}$ & $\begin{array}{c}-1.700^{\star \star \star} \\
(0.000)\end{array}$ & $\begin{array}{r}-0.692^{\star} \\
(0.039)\end{array}$ \\
\hline Religiosity & $\begin{array}{c}-0.101 \\
(0.052)\end{array}$ & $\begin{array}{r}-0.108^{\star} \\
(0.034)\end{array}$ & $\begin{array}{c}-0.184^{\star \star \star} \\
(0.000)\end{array}$ & $\begin{array}{c}-0.089 \\
(0.088)\end{array}$ & $\begin{array}{c}-0.022 \\
(0.675)\end{array}$ & $\begin{array}{c}0.015 \\
(0.777)\end{array}$ & $\begin{array}{c}0.123^{*} \\
(0.018)\end{array}$ \\
\hline Female & $\begin{array}{c}-0.114 \\
(0.440)\end{array}$ & $\begin{array}{c}-0.786^{\star \star \star} \\
(0.000)\end{array}$ & $\begin{array}{c}-0.216 \\
(0.152)\end{array}$ & $\begin{array}{c}0.009 \\
(0.953)\end{array}$ & $\begin{array}{c}-0.103 \\
(0.485)\end{array}$ & $\begin{array}{c}0.344^{\star} \\
(0.022)\end{array}$ & $\begin{array}{c}-0.250 \\
(0.119)\end{array}$ \\
\hline Black & $\begin{array}{c}0.034 \\
(0.848)\end{array}$ & $\begin{array}{c}-0.237 \\
(0.159)\end{array}$ & $\begin{array}{c}-0.005 \\
(0.976)\end{array}$ & $\begin{array}{c}-0.087 \\
(0.602)\end{array}$ & $\begin{array}{c}-0.187 \\
(0.265)\end{array}$ & $\begin{array}{c}-0.112 \\
(0.531)\end{array}$ & $\begin{array}{c}0.042 \\
(0.817)\end{array}$ \\
\hline Hispanic & $\begin{array}{c}0.215 \\
(0.263)\end{array}$ & $\begin{array}{c}0.171 \\
(0.333)\end{array}$ & $\begin{array}{c}0.138 \\
(0.427)\end{array}$ & $\begin{array}{c}0.186 \\
(0.336)\end{array}$ & $\begin{array}{c}0.325 \\
(0.096)\end{array}$ & $\begin{array}{c}0.285 \\
(0.117)\end{array}$ & $\begin{array}{c}0.103 \\
(0.609)\end{array}$ \\
\hline Rural & $\begin{array}{c}0.058^{\star} \\
(0.018)\end{array}$ & $\begin{array}{c}0.043 \\
(0.085)\end{array}$ & $\begin{array}{c}0.038 \\
(0.088)\end{array}$ & $\begin{array}{r}0.061^{*} \\
(0.012)\end{array}$ & $\begin{array}{l}0.086^{\star * *} \\
(0.001)\end{array}$ & $\begin{array}{l}0.087^{\star \star} \\
(0.004)\end{array}$ & $\begin{array}{l}0.080^{\star \star \star} \\
(0.001)\end{array}$ \\
\hline Urban & $\begin{array}{c}-0.000 \\
(0.121)\end{array}$ & $\begin{array}{c}-0.000 \\
(0.124)\end{array}$ & $\begin{array}{r}-0.000 \\
(0.116)\end{array}$ & $\begin{array}{r}-0.000^{\star} \\
(0.047)\end{array}$ & $\begin{array}{l}-0.001^{\star \star \star} \\
(0.001)\end{array}$ & $\begin{array}{r}-0.001^{*} \\
(0.013)\end{array}$ & $\begin{array}{c}-0.001^{\star \star \star} \\
(0.001)\end{array}$ \\
\hline Age & $\begin{array}{c}0.212 \\
(0.541)\end{array}$ & $\begin{array}{r}-0.296 \\
(0.358)\end{array}$ & $\begin{array}{c}-0.005 \\
(0.976)\end{array}$ & $\begin{array}{c}0.276 \\
(0.296)\end{array}$ & $\begin{array}{c}0.081 \\
(0.826)\end{array}$ & $\begin{array}{c}0.419 \\
(0.213)\end{array}$ & $\begin{array}{c}0.449 \\
(0.278)\end{array}$ \\
\hline Age squared & $\begin{array}{c}0.798^{*} \\
(0.034)\end{array}$ & $\begin{array}{c}0.072 \\
(0.840)\end{array}$ & $\begin{array}{c}0.138 \\
(0.427)\end{array}$ & $\begin{array}{c}0.630^{\star} \\
(0.040)\end{array}$ & $\begin{array}{c}-0.112 \\
(0.783)\end{array}$ & $\begin{array}{c}0.257 \\
(0.492)\end{array}$ & $\begin{array}{c}0.521 \\
(0.224)\end{array}$ \\
\hline Political knowledge & $\begin{array}{c}0.839^{\star} \\
(0.028)\end{array}$ & $\begin{array}{c}-0.151 \\
(0.690)\end{array}$ & $\begin{array}{c}0.038 \\
(0.088)\end{array}$ & $\begin{array}{c}0.610 \\
(0.054)\end{array}$ & $\begin{array}{c}0.057 \\
(0.892)\end{array}$ & $\begin{array}{c}0.535 \\
(0.161)\end{array}$ & $\begin{array}{c}-0.003 \\
(0.994)\end{array}$ \\
\hline Employer-sponsored & $\begin{array}{c}0.017 \\
(0.747)\end{array}$ & $\begin{array}{c}-0.204^{\star \star *} \\
(0.000)\end{array}$ & $\begin{array}{c}-0.069 \\
(0.201)\end{array}$ & $\begin{array}{c}0.068 \\
(0.262)\end{array}$ & $\begin{array}{c}0.006 \\
(0.922)\end{array}$ & $\begin{array}{c}0.010 \\
(0.863)\end{array}$ & $\begin{array}{c}0.059 \\
(0.280)\end{array}$ \\
\hline
\end{tabular}


Table 1b. (Continued)

\begin{tabular}{|c|c|c|c|c|c|c|c|}
\hline & (1) & $(2)$ & (3) & (4) & (5) & (6) & (7) \\
\hline Variables & Good Samaritan & Safe Sites & Test Kits & Diversion & Medicaid & Insurance & Marijuana \\
\hline Medicare & $\begin{array}{c}0.048 \\
(0.793)\end{array}$ & $\begin{array}{c}-0.197 \\
(0.297)\end{array}$ & $\begin{array}{c}-0.206 \\
(0.225)\end{array}$ & $\begin{array}{c}-0.267 \\
(0.170)\end{array}$ & $\begin{array}{c}-0.061 \\
(0.755)\end{array}$ & $\begin{array}{c}0.143 \\
(0.428)\end{array}$ & $\begin{array}{c}-0.210 \\
(0.248)\end{array}$ \\
\hline Constant & $\begin{array}{c}-0.243 \\
(0.277)\end{array}$ & $\begin{array}{c}-0.008 \\
(0.971)\end{array}$ & $\begin{array}{c}0.021 \\
(0.920)\end{array}$ & $\begin{array}{c}-0.324 \\
(0.106)\end{array}$ & $\begin{array}{c}-0.205 \\
(0.373)\end{array}$ & $\begin{array}{c}-0.134 \\
(0.551)\end{array}$ & $\begin{array}{c}-0.150 \\
(0.500)\end{array}$ \\
\hline Education & Yes & Yes & Yes & Yes & Yes & Yes & Yes \\
\hline Income & Yes & Yes & Yes & Yes & Yes & Yes & Yes \\
\hline Mckelvey and Zavoina $\mathrm{R}^{2}$ & 0.178 & 0.266 & 0.118 & 0.179 & 0.245 & 0.232 & 0.220 \\
\hline Observations & 1,329 & 1,328 & 1,320 & 1,321 & 1,324 & 1,323 & 1,319 \\
\hline
\end{tabular}

Robust $p$-values in parentheses ${ }^{\star * \star} p<0.001$, ** $p<0.01,{ }^{*} p<0.05$. 


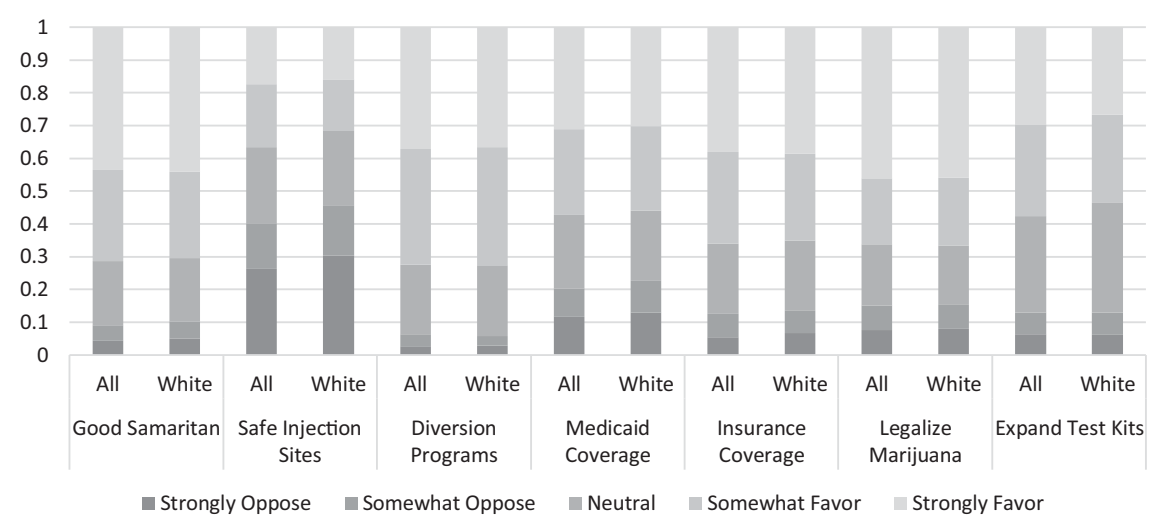

Figure 1. Average predictions for models from Tables $1 \mathrm{a}$ and $1 \mathrm{~b}$.

successful example for respondents to consider, and only a few exist in North America, in Canada. Second, the stigmatisation of individuals who use injection drugs influences support for SIFS (McGinty et al. 2018). Some individuals might simply be uncomfortable providing a space for individuals to use drugs in violation of the law. In sum, Americans appear quite favourable of policies supporting individuals suffering from OUD with the exceptions of SIFS and, to a lesser degree, drug test kits.

Next, we present the AMEs for the five explanatory variables of interest we hypothesised about (Figure 2). We hold all variables constant for the three indicator variables (personal choice, personal connection and addiction) while varying the respective explanatory variable between the two choices. We only display results for those changes in AMEs that were statistically significant, at least at the 0.1 level.

Comparing those who think that OUD is a personal choice to those who think it is due to factors beyond the individual's control shows consistently significant effects. For five policies (all except SIFS and drug testing kits), we see increases in the strongly and somewhat oppose categories as well as the neutral category. In contrast, the strongly favourable category is markedly reduced for those who consider addiction a Personal Choice. Personal Connections, on the other hand, appear to have the opposite effect. That is, there appears to be a significant decrease in the oppositional and neutral categories paired with a significant increase in the strongly favourable bin for those with personal connections as compared to those without. While we see no effect on the SIFS policy option, we see some limited effects for the drug testing kit option. The most pronounced effect can be found comparing those suffering from OUD to those who do not. Here, increases in the strongly favourable category exceed 0.300 in two of the seven cases and 0.200 in five of the seven cases. Notably, we also see an effect for SIFS (0.108), albeit the smallest effect among the policy choices.

For the remaining two key explanatory variables, Ideology and Racial Resentment, we vary the variables from their minimum to their maximum value while holding all other variables constant. Again, we only display results for those changes in AMEs 
that were statistically significant, at least at the 0.1 level. Varying the Ideology of respondents from extremely liberal to extremely conservative has a ubiquitous effect on all policy options and all answer categories. Comparing the most liberal to the most conservative respondents illustrates a significant reduction in the most favourable category for all seven policy options with increases in both oppositional and the neutral category. For SIFS, substantial reductions are also visible in the somewhat favourable category, and the increase in the strongly opposed category approaches 0.400. Finally, when varying Racial Resentment from high to low, the effects are even more pronounced, approaching 0.500 .

\section{Policies designed to punish individuals for OUD}

We also asked respondents their preferences about policies designed to punish those suffering from OUD. These include jail time for those who misuse opioids (Table 2: Model 1), denying social welfare benefits to those who test positive for drugs (Table 2: Model 2), charging parents addicted to drugs with child abuse (Table 2: Model 3) and charging pregnant mothers suffering from OUD with child abuse (Table 2: Model 4). Again, we estimate all models for all respondents (Table $2 \mathrm{a}$ ) and non-Hispanic Whites (Table $2 \mathrm{~b}$ ), respectively.

Again, as coefficients from ordered logit models are limited in their interpretative value we hence rely once more on graphical displays to illustrate our findings. Figure 3 shows the average predictions for the estimates presented in Tables $2 \mathrm{a}$ and $2 \mathrm{~b}$. For three of the four policy options, the somewhat and strongly favourable categories exceed 0.500 , with the remaining policy option requiring jail time for individuals who misuse opioids, almost approaching that mark. On the other end of the spectrum, the two oppositional categories generally combine for $0.150-0.200$, with the jail category again serving as an exception with almost 0.300. Figures 4a-e corresponds to Figure 2a-e and display the AMEs for the punitive policy options.

We find consistent effects for our Personal Choice variable. That is, those who think that OUD is a personal choice are much more likely to be supportive of all four punitive policies compared to those who think OUD is related to factors outside an individual's control. We note that the size of the effect is smaller than for supportive policies. Our findings for personal connections are more complex. While those with a personal connection are generally less favourable of requiring jail time for those with OUD, we also note significant increases in support for pursuing criminal charges against parents and pregnant women. Generally, we find only limited effects for punitive policies for those suffering from OUD themselves: they are significantly less supportive of requiring jail sentences and other criminal persecutions of all types. With the exceptions of requiring jail time, the effects are only significant for the strongly favourable category, and they are generally substantively small.

We find more consistent and substantively large effects for Ideology and Racial Resentment. As conservatism increases, so does support for punitive policies. Notably, this effect is largest for reducing welfare benefits followed by punishment for pregnant women with OUD issues and requiring jail time. We do not find statistically significant effects for the prosecution of parents' policy option. However, 
Table 2a. Punitive policies, all respondents

\begin{tabular}{|c|c|c|c|c|}
\hline & (1) & (2) & (3) & (4) \\
\hline Variables & Jailtime & Benefits reduction & Arrest parents & Arrest pregnant women \\
\hline Personal choice & $\begin{array}{l}0.497^{\star * *} \\
(0.000)\end{array}$ & $\begin{array}{l}0.560^{\star * \star} \\
(0.000)\end{array}$ & $\begin{array}{l}0.330^{\star *} \\
(0.004)\end{array}$ & $\begin{array}{c}0.253^{*} \\
(0.033)\end{array}$ \\
\hline Opioid use disorder & $\begin{array}{r}-1.043^{\star} \\
(0.010)\end{array}$ & $\begin{array}{l}-0.387 \\
(0.290)\end{array}$ & $\begin{array}{c}-0.668 \\
(0.105)\end{array}$ & $\begin{array}{l}-0.116 \\
(0.722)\end{array}$ \\
\hline Personal connection & $\begin{array}{r}-0.273^{\star} \\
(0.015)\end{array}$ & $\begin{array}{c}0.162 \\
(0.162)\end{array}$ & $\begin{array}{c}0.212 \\
(0.067)\end{array}$ & $\begin{array}{l}0.364^{\star \star} \\
(0.002)\end{array}$ \\
\hline Ideology & $\begin{array}{l}0.116^{\star *} \\
(0.001)\end{array}$ & $\begin{array}{l}0.213^{\star \star \star} \\
(0.000)\end{array}$ & $\begin{array}{c}0.045 \\
(0.179)\end{array}$ & $\begin{array}{l}0.119^{\star \star \star} \\
(0.001)\end{array}$ \\
\hline Religiosity & $\begin{array}{l}-0.249^{\star \star \star *} \\
(0.000)\end{array}$ & $\begin{array}{c}-0.159^{\star \star \star} \\
(0.000)\end{array}$ & $\begin{array}{c}-0.163^{\star \star \star} \\
(0.000)\end{array}$ & $\begin{array}{c}-0.138^{\star \star \star} \\
(0.001)\end{array}$ \\
\hline Female & $\begin{array}{c}0.014 \\
(0.904)\end{array}$ & $\begin{array}{c}0.211 \\
(0.091)\end{array}$ & $\begin{array}{c}0.014 \\
(0.912)\end{array}$ & $\begin{array}{c}0.013 \\
(0.915)\end{array}$ \\
\hline Black & $\begin{array}{r}-0.367^{\star} \\
(0.042)\end{array}$ & $\begin{array}{c}-0.478^{\star *} \\
(0.004)\end{array}$ & $\begin{array}{c}-0.331 \\
(0.071)\end{array}$ & $\begin{array}{r}-0.404^{*} \\
(0.022)\end{array}$ \\
\hline Hispanic & $\begin{array}{c}-0.147 \\
(0.372)\end{array}$ & $\begin{array}{c}0.158 \\
(0.395)\end{array}$ & $\begin{array}{c}0.042 \\
(0.822)\end{array}$ & $\begin{array}{c}-0.181 \\
(0.351)\end{array}$ \\
\hline Rural & $\begin{array}{l}0.489^{\star \star \star} \\
(0.000)\end{array}$ & $\begin{array}{c}0.256 \\
(0.071)\end{array}$ & $\begin{array}{c}0.244 \\
(0.101)\end{array}$ & $\begin{array}{c}0.229 \\
(0.102)\end{array}$ \\
\hline Urban & $\begin{array}{c}0.110 \\
(0.428)\end{array}$ & $\begin{array}{c}0.158 \\
(0.253)\end{array}$ & $\begin{array}{c}0.034 \\
(0.808)\end{array}$ & $\begin{array}{c}0.014 \\
(0.920)\end{array}$ \\
\hline Age & $\begin{array}{c}0.010 \\
(0.555)\end{array}$ & $\begin{array}{r}0.045^{\star} \\
(0.016)\end{array}$ & $\begin{array}{c}-0.009 \\
(0.629)\end{array}$ & $\begin{array}{l}0.050^{\star *} \\
(0.009)\end{array}$ \\
\hline Age squared & $\begin{array}{c}-0.000 \\
(0.403)\end{array}$ & $\begin{array}{c}-0.000^{\star} \\
(0.037)\end{array}$ & $\begin{array}{c}0.000 \\
(0.529)\end{array}$ & $\begin{array}{c}-0.001^{\star \star} \\
(0.005)\end{array}$ \\
\hline Political knowledge & $\begin{array}{c}-0.217^{\star \star \star} \\
(0.000)\end{array}$ & $\begin{array}{l}-0.158^{\star \star \star} \\
(0.000)\end{array}$ & $\begin{array}{r}-0.099^{\star} \\
(0.018)\end{array}$ & $\begin{array}{r}-0.059 \\
(0.179)\end{array}$ \\
\hline Employer-sponsored & $\begin{array}{c}-0.046 \\
(0.745)\end{array}$ & $\begin{array}{c}0.167 \\
(0.269)\end{array}$ & $\begin{array}{c}0.032 \\
(0.812)\end{array}$ & $\begin{array}{c}0.192 \\
(0.178)\end{array}$ \\
\hline Medicare & $\begin{array}{c}0.092 \\
(0.543)\end{array}$ & $\begin{array}{c}0.091 \\
(0.591)\end{array}$ & $\begin{array}{c}0.000 \\
(0.998)\end{array}$ & $\begin{array}{c}0.211 \\
(0.207)\end{array}$ \\
\hline Education & Yes & Yes & Yes & Yes \\
\hline $\begin{array}{l}\text { Income } \\
\text { McKelvey and Zavoina } \mathrm{R}^{2}\end{array}$ & $\begin{array}{r}\text { Yes } \\
0.152\end{array}$ & $\begin{array}{r}\text { Yes } \\
0.149\end{array}$ & $\begin{array}{r}\text { Yes } \\
0.077\end{array}$ & $\begin{array}{r}\text { Yes } \\
0.091\end{array}$ \\
\hline Observations & 1,998 & 2,004 & 2,003 & 2,005 \\
\hline
\end{tabular}

Robust $p$-values in parentheses ${ }^{* * *} p<0.001,{ }^{* *} p<0.01,{ }^{*} p<0.05$.

the most pronounced effects are apparent comparing those with low levels of racial resentment to those with high levels. The order of effect sizes mirrors that of ideology and ranges from almost 0.600 for eliminating welfare benefits and 0.500 for arresting pregnant women to about 0.300 for jail time and arresting of parents.

\section{Overall support for supportive and punitive policies}

Finally, we created several indices that combine all seven supportive policy options and all four punitive policy options, respectively. The first index provides a count of the number of policies (supportive or punitive) that each individual is somewhat or strongly favourable towards, while the second for each type of policy is an additive index that adds the ordinal value of the responses (from strongly oppose coded as 1 to strong favour coded as 5). All indices have Cronbach's alpha values of around 
Table 2b. Punitive policies, White non-Hispanics only

\begin{tabular}{|c|c|c|c|c|}
\hline & (1) & (2) & (3) & (4) \\
\hline Variables & Jailtime & Benefits reduction & Arrest parents & Arrest pregnant women \\
\hline Personal choice & $\begin{array}{l}0.439^{\star *} \\
(0.003)\end{array}$ & $\begin{array}{l}0.427^{\star \star} \\
(0.004)\end{array}$ & $\begin{array}{c}0.232 \\
(0.109)\end{array}$ & $\begin{array}{c}0.125 \\
(0.414)\end{array}$ \\
\hline Opioid use disorder & $\begin{array}{c}-1.025 \\
(0.055)\end{array}$ & $\begin{array}{c}0.191 \\
(0.675)\end{array}$ & $\begin{array}{c}-0.698 \\
(0.206)\end{array}$ & $\begin{array}{c}0.033 \\
(0.933)\end{array}$ \\
\hline Personal connection & $\begin{array}{c}-0.176 \\
(0.208)\end{array}$ & $\begin{array}{c}0.141 \\
(0.330)\end{array}$ & $\begin{array}{c}0.143 \\
(0.299)\end{array}$ & $\begin{array}{l}0.491^{\star \star \star} \\
(0.001)\end{array}$ \\
\hline Ideology & $\begin{array}{c}0.060 \\
(0.231)\end{array}$ & $\begin{array}{c}0.111^{*} \\
(0.025)\end{array}$ & $\begin{array}{c}-0.065 \\
(0.172)\end{array}$ & $\begin{array}{c}-0.048 \\
(0.296)\end{array}$ \\
\hline Racial resentment & $\begin{array}{l}2.066^{\star \star \star} \\
(0.000)\end{array}$ & $\begin{array}{l}3.318^{\star \star \star} \\
(0.000)\end{array}$ & $\begin{array}{l}1.794^{\star \star *} \\
(0.000)\end{array}$ & $\begin{array}{l}2.758^{\star \star \star} \\
(0.000)\end{array}$ \\
\hline Religiosity & $\begin{array}{l}-0.180^{* * *} \\
(0.000)\end{array}$ & $\begin{array}{c}-0.136^{\star \star} \\
(0.007)\end{array}$ & $\begin{array}{r}-0.112^{*} \\
(0.022)\end{array}$ & $\begin{array}{c}-0.116^{*} \\
(0.017)\end{array}$ \\
\hline Female & $\begin{array}{c}-0.090 \\
(0.537)\end{array}$ & $\begin{array}{c}0.173 \\
(0.261)\end{array}$ & $\begin{array}{c}-0.009 \\
(0.951)\end{array}$ & $\begin{array}{c}-0.044 \\
(0.780)\end{array}$ \\
\hline Rural & $\begin{array}{l}0.473^{\star *} \\
(0.004)\end{array}$ & $\begin{array}{c}0.276 \\
(0.099)\end{array}$ & $\begin{array}{c}0.259 \\
(0.133)\end{array}$ & $\begin{array}{c}0.220 \\
(0.188)\end{array}$ \\
\hline Urban & $\begin{array}{c}0.160 \\
(0.400)\end{array}$ & $\begin{array}{c}0.304 \\
(0.101)\end{array}$ & $\begin{array}{c}0.040 \\
(0.844)\end{array}$ & $\begin{array}{c}0.119 \\
(0.530)\end{array}$ \\
\hline Age & $\begin{array}{c}-0.030 \\
(0.210)\end{array}$ & $\begin{array}{c}0.006 \\
(0.806)\end{array}$ & $\begin{array}{c}-0.026 \\
(0.263)\end{array}$ & $\begin{array}{c}0.006 \\
(0.810)\end{array}$ \\
\hline Age squared & $\begin{array}{c}0.000 \\
(0.365)\end{array}$ & $\begin{array}{c}-0.000 \\
(0.965)\end{array}$ & $\begin{array}{c}0.000 \\
(0.165)\end{array}$ & $\begin{array}{c}-0.000 \\
(0.629)\end{array}$ \\
\hline Political knowledge & $\begin{array}{c}-0.202^{\star \star \star} \\
(0.000)\end{array}$ & $\begin{array}{c}-0.162^{\star \star} \\
(0.004)\end{array}$ & $\begin{array}{c}-0.146^{\star \star} \\
(0.007)\end{array}$ & $\begin{array}{c}-0.050 \\
(0.366)\end{array}$ \\
\hline Employer-sponsored & $\begin{array}{c}0.348 \\
(0.065)\end{array}$ & $\begin{array}{c}0.332 \\
(0.081)\end{array}$ & $\begin{array}{c}0.396^{\star} \\
(0.024)\end{array}$ & $\begin{array}{l}0.655^{\star \star \star} \\
(0.000)\end{array}$ \\
\hline Medicare & $\begin{array}{c}0.218 \\
(0.299)\end{array}$ & $\begin{array}{c}-0.012 \\
(0.960)\end{array}$ & $\begin{array}{c}0.112 \\
(0.605)\end{array}$ & $\begin{array}{c}0.389 \\
(0.086)\end{array}$ \\
\hline Education & Yes & Yes & Yes & Yes \\
\hline Income & Yes & Yes & Yes & Yes \\
\hline McKelvey and Zavoina $\mathrm{R}^{2}$ & 0.218 & 0.290 & 0.128 & 0.191 \\
\hline Observations & 1,321 & 1,326 & 1,324 & 1,325 \\
\hline
\end{tabular}

Robust $\mathrm{p}$-values in parentheses ${ }^{\star \star \star} \mathrm{p}<0.001$, ${ }^{\star \star} \mathrm{p}<0.01,{ }^{\star} \mathrm{p}<0.05$.

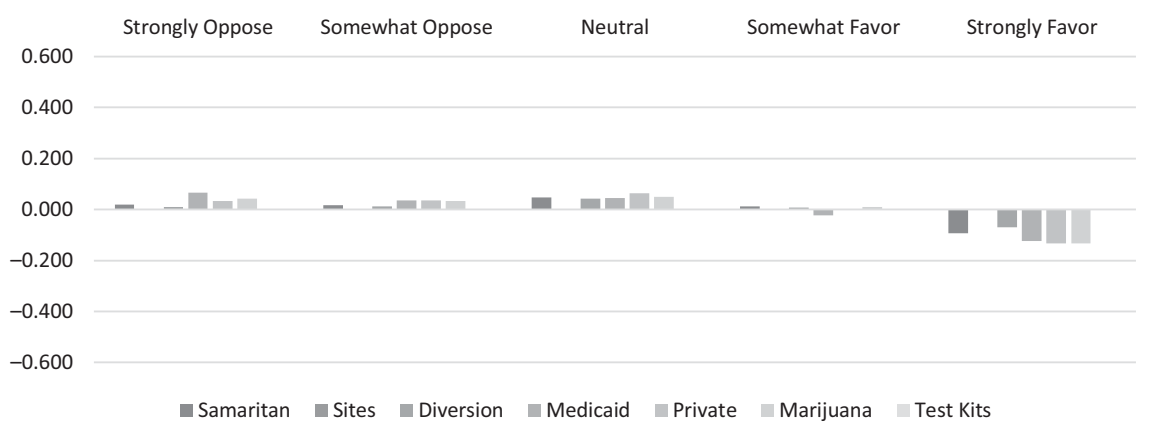

Figure 2a. Average marginal effects for personal choice. 


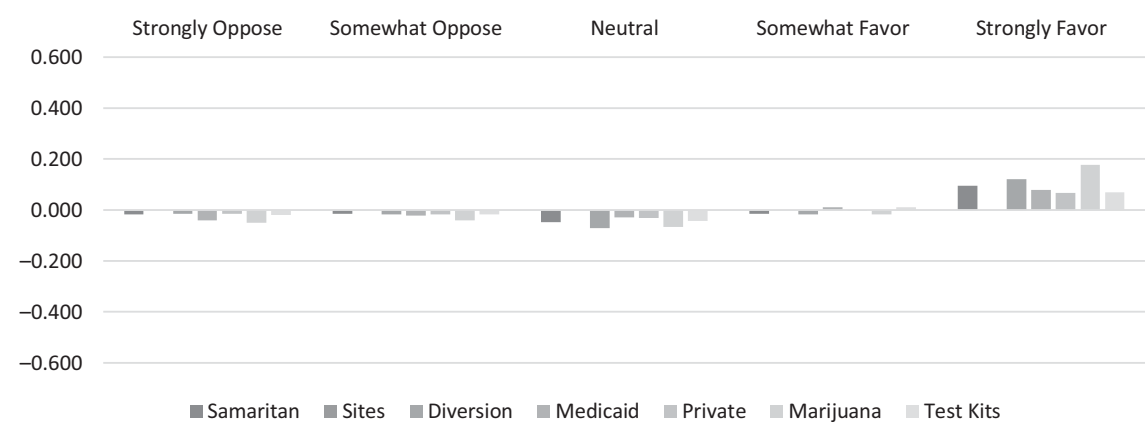

Figure 2b. Average marginal effects for personal connection.

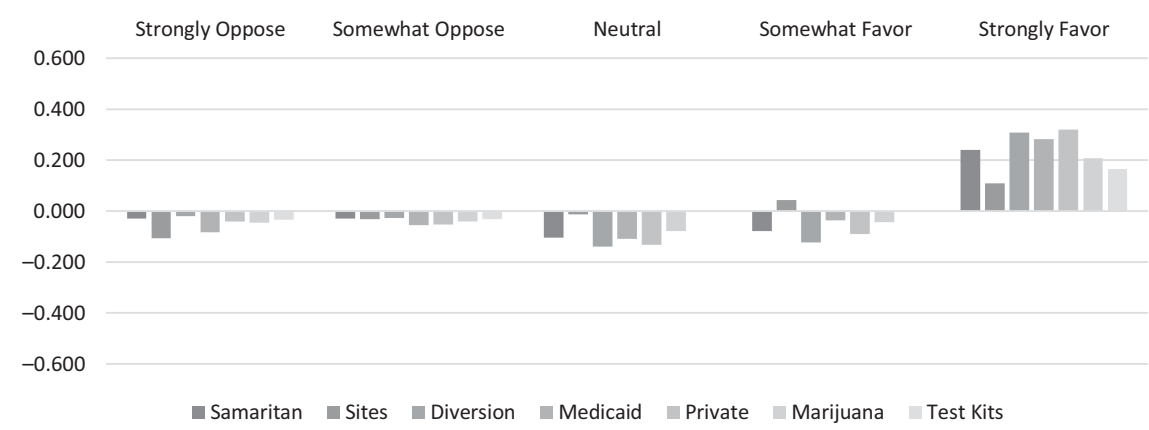

Figure 2c. Average marginal effects for opioid use disorder.

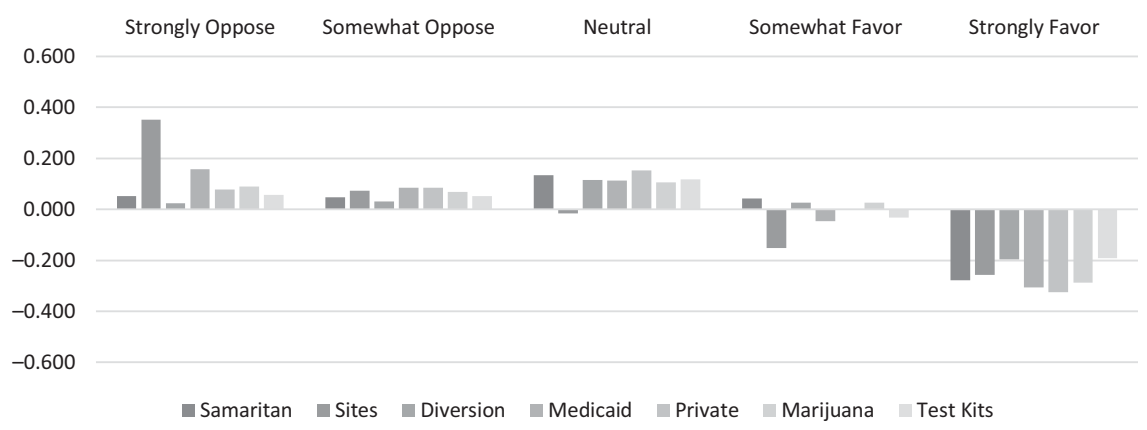

Figure 2d. Average marginal effects for ideology.

0.800 , indicating appropriate levels of reliability. We then utilised the index values as dependent variables and estimated both Poisson and standard OLS models utilising the same independent variables as in previous models. For both supportive and punitive indices, our findings are analogous for the respective indices, and we thus 


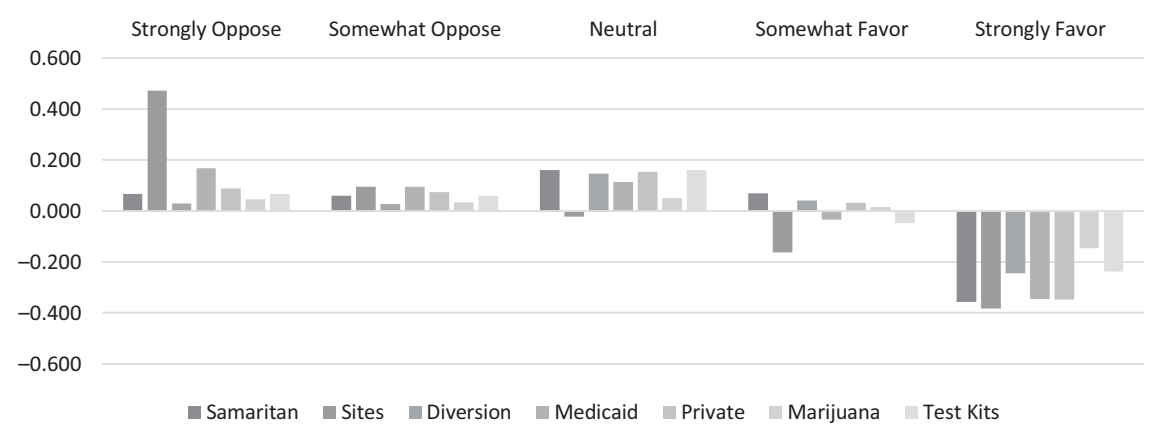

Figure 2e. Average marginal effects for racial resentment.

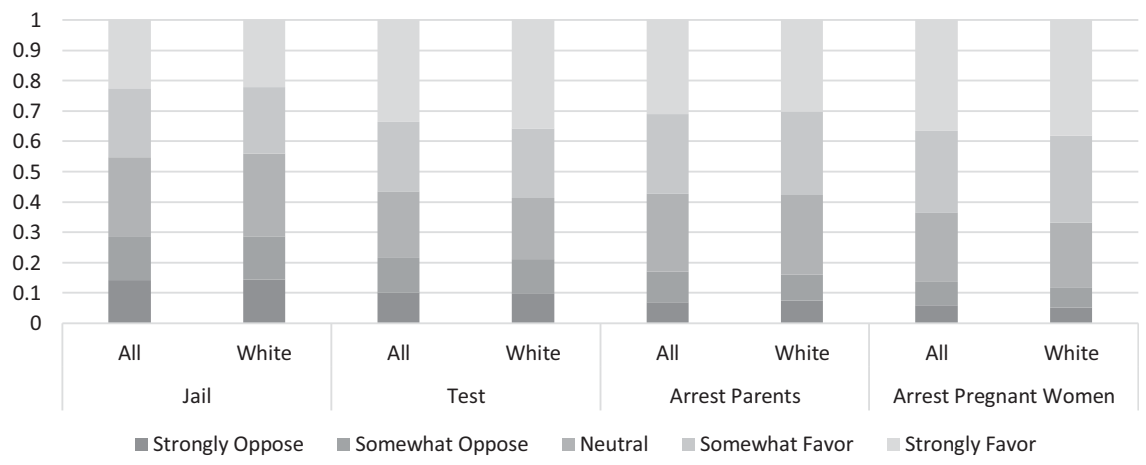

Figure 3. Average predictions for models from Tables $2 a$ and $2 b$.

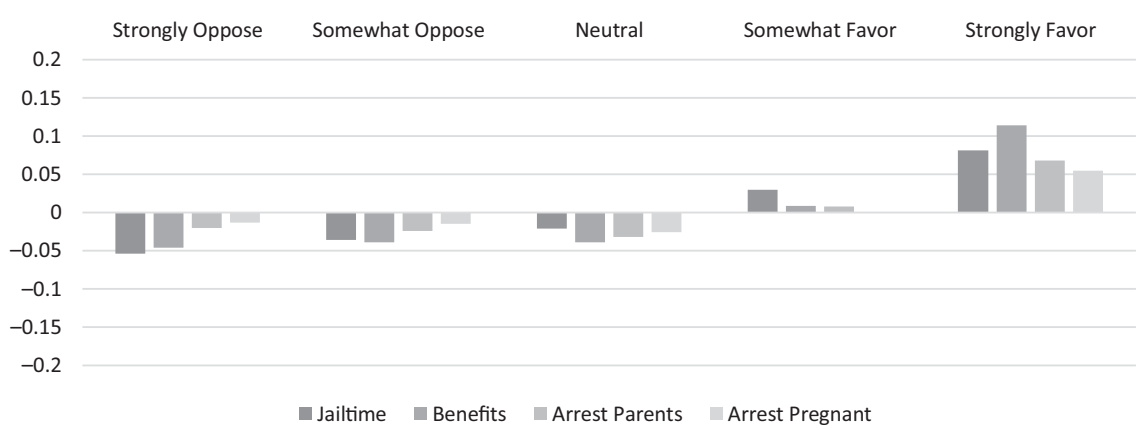

Figure 4a. Average marginal effects for personal choice.

focus on the first set of indices described above. We focus on the OLS models (Table 3) to present our findings below (Poisson models omitted) for ease of interpretation. 


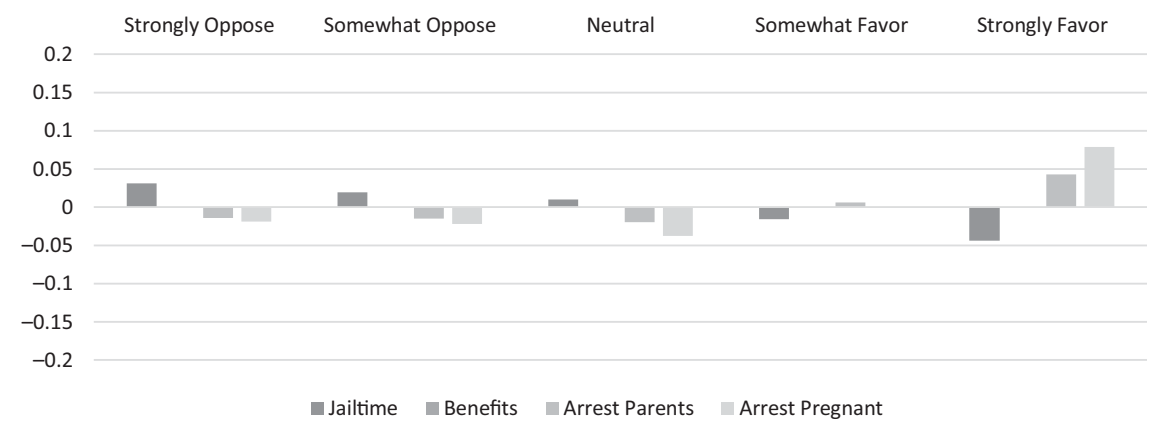

Figure 4b. Average marginal effects for personal connection.

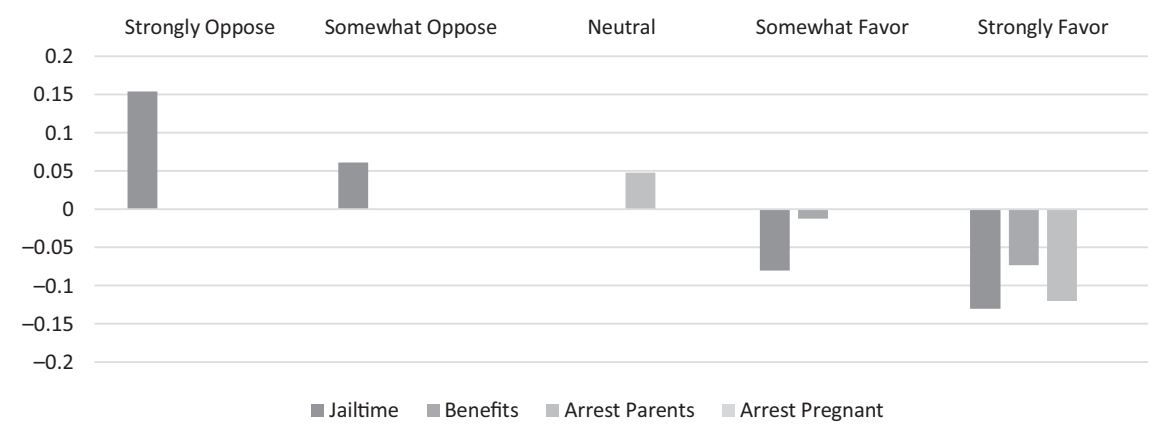

Figure 4c. Average marginal effects for opioid use disorder.

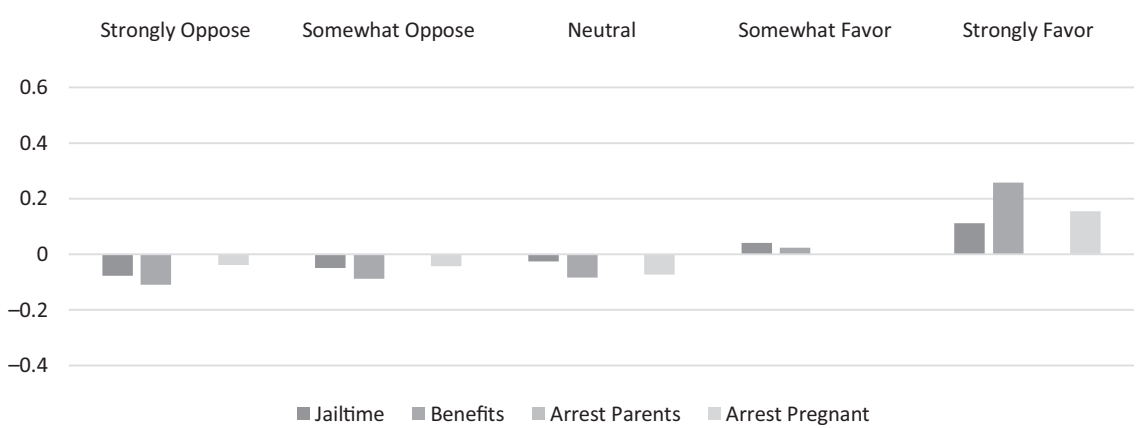

Figure 4d. Average marginal effects for ideology.

The findings for the supportive-policies indices are presented in Table 3 above for OLS models. Looking at Supportive Index 1 for all respondents, respondents who viewed OUD as a Personal Choice supported 0.5 fewer policies (out of 7). Similarly, each increase in the level of conservatism was associated with a decrease in support by 0.3 policies. Support for more policies was related to having a Personal 


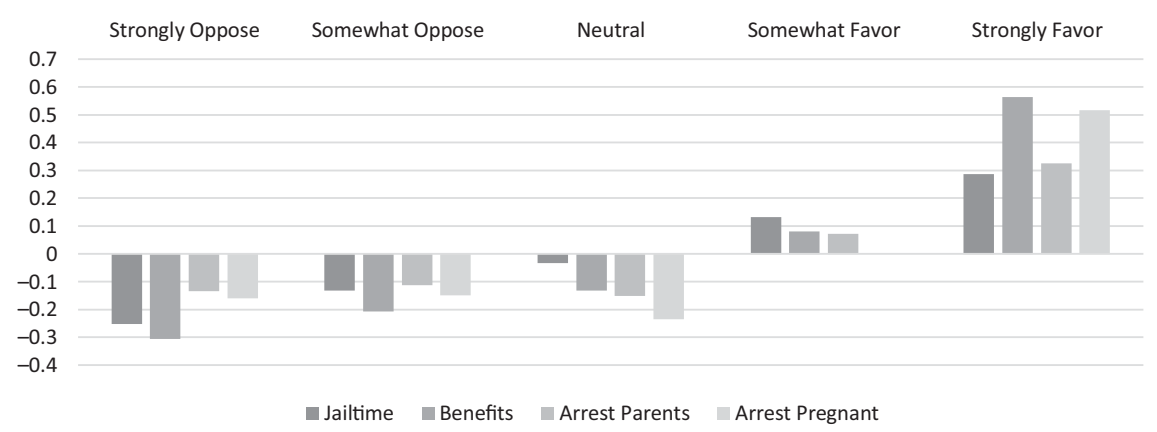

Figure 4e. Average marginal effects for racial resentment.

Connection (0.8 more policies) and personal experience with OUD (1.1 more policies). Findings for non-Hispanic White respondents are analogous. That is, those who consider OUD a Personal Choice and conservatives support fewer policies ( 0.4 for the former and 0.2 for each increase in the level of the latter) while those with Personal Connections (0.8) and those with personal experiences with OUD (1.5) support more policies. However, the effect of Racial Resentment once more stands out: respondents with high levels of Racial Resentment support almost two fewer policies compared to those with low levels of resentment.

For Punitive-Policies Index 1, also presented in Table 3, we find that considering OUD a Personal Choice is associated with support for an additional 0.3 policies (out of four). In comparison, each increasing level of conservatism is associated with support for an additional 0.1 policies supported among all respondents. We also find effects for Personal Connection. Individuals who know someone suffering from OUD support 0.2 more punitive policies. We do not find any effects for personal experience with $O U D$, which is just above our cut-off p-value of 0.05 . These findings are robust when only analysing non-Hispanic Whites for the Personal Connection, personal experience with OUD, and Personal Choice variables while we find no effect for Ideology. However, we note the again strong effect for Racial Resentment: those with high levels of resentment support 2 more punitive policies compared to those with low levels.

\section{Discussion}

Our research applies Weiner's attribution theory of controllability to individuals' attitudes about the causes of OUD. According to attribution theory, the causes of addiction are perceived to be either controllable (driven by personal choice) or not controllable (driven by situational factors). We posited that attributions and other factors like personal connection, personal experience with addiction, ideology and racial resentment would influence how individuals view policies designed to combat the opioid epidemic.

Consistent with our theoretical expectations, we find significant, and at times substantial, differences in support for policies to combat the opioid epidemic across our variables of interest. Whereas a personal connection and personal experience 
Table 3. Results for analyses of indices of supportive and punitive policies

\begin{tabular}{|c|c|c|c|c|c|c|c|c|}
\hline \multirow[b]{3}{*}{ Variables } & \multicolumn{2}{|c|}{ Supportive-policies index 1} & \multicolumn{2}{|c|}{ Supportive-policies index 2} & \multicolumn{2}{|c|}{ Punitive-policies index 1} & \multirow{2}{*}{\multicolumn{2}{|c|}{ Punitive-policies index 2}} \\
\hline & All & Non-White Hispanics & All & Non-White Hispanics & All & Non-White Hispanics & & \\
\hline & OLS & OLS & OLS & OLS & OLS & OLS & All & Non-White Hispanics \\
\hline Personal choice & $\begin{array}{c}-0.461^{\star \star \star} \\
(0.00)\end{array}$ & $\begin{array}{c}-0.413^{\star *} \\
(0.006)\end{array}$ & $\begin{array}{c}-1.454^{\star \star \star} \\
(0.000)\end{array}$ & $\begin{array}{c}-1.139^{\star *} \\
(0.003)\end{array}$ & $\begin{array}{l}0.344^{\star \star \star} \\
(0.000)\end{array}$ & $\begin{array}{c}0.219^{\star} \\
(0.044)\end{array}$ & $\begin{array}{l}1.158^{\star \star \star} \\
(0.000)\end{array}$ & $\begin{array}{l}0.860^{\star \star \star} \\
(0.001)\end{array}$ \\
\hline Opioid use disorder & $\begin{array}{l}1.139^{\star \star *} \\
(0.000)\end{array}$ & $\begin{array}{l}1.479^{\star \star \star} \\
(0.000)\end{array}$ & $\begin{array}{l}3.444^{\star * \star} \\
(0.000)\end{array}$ & $\begin{array}{l}4.014^{\star \star \star} \\
(0.000)\end{array}$ & $\begin{array}{c}-0.358 \\
(0.078)\end{array}$ & $\begin{array}{c}-0.202 \\
(0.443)\end{array}$ & $\begin{array}{r}-1.521^{\star} \\
(0.019)\end{array}$ & $\begin{array}{c}-1.054 \\
(0.198)\end{array}$ \\
\hline Personal connection & $\begin{array}{l}0.756^{\star \star \star} \\
(0.000)\end{array}$ & $\begin{array}{l}0.847^{\star \star \star} \\
(0.000)\end{array}$ & $\begin{array}{l}1.468^{\star \star \star} \\
(0.000)\end{array}$ & $\begin{array}{l}1.674^{\star \star \star} \\
(0.000)\end{array}$ & $\begin{array}{c}0.200^{*} \\
(0.020)\end{array}$ & $\begin{array}{c}0.237^{\star} \\
(0.018)\end{array}$ & $\begin{array}{c}0.094 \\
(0.676)\end{array}$ & $\begin{array}{c}0.160 \\
(0.526)\end{array}$ \\
\hline Ideology & $\begin{array}{c}-0.319^{\star \star \star} \\
(0.000)\end{array}$ & $\begin{array}{c}-0.229^{\star \star \star} \\
(0.000)\end{array}$ & $\begin{array}{c}0.031 \\
(0.935)\end{array}$ & $\begin{array}{c}-0.280 \\
(0.538)\end{array}$ & $\begin{array}{c}0.140 \\
(0.182)\end{array}$ & $\begin{array}{l}0.398^{\star *} \\
(0.002)\end{array}$ & $\begin{array}{l}0.346^{\star \star \star} \\
(0.000)\end{array}$ & $\begin{array}{c}0.097 \\
(0.238)\end{array}$ \\
\hline Racial resentment & & $\begin{array}{c}-1.848^{\star \star \star} \\
(0.000)\end{array}$ & $\begin{array}{c}-0.362 \\
(0.429)\end{array}$ & $\begin{array}{c}-0.439 \\
(0.417)\end{array}$ & $\begin{array}{c}0.208 \\
(0.119)\end{array}$ & $\begin{array}{c}0.213 \\
(0.191)\end{array}$ & & $\begin{array}{l}5.582^{\star \star \star *} \\
(0.000)\end{array}$ \\
\hline Religiosity & $\begin{array}{r}-0.085^{\star} \\
(0.046)\end{array}$ & $\begin{array}{c}-0.057 \\
(0.238)\end{array}$ & $\begin{array}{c}-0.984^{\star \star \star} \\
(0.000)\end{array}$ & $\begin{array}{c}-0.731^{\star \star \star} \\
(0.000)\end{array}$ & $\begin{array}{l}0.090^{\star \star \star} \\
(0.000)\end{array}$ & $\begin{array}{c}0.010 \\
(0.747)\end{array}$ & $\begin{array}{c}-0.476^{\star \star \star} \\
(0.000)\end{array}$ & $\begin{array}{c}-0.376^{\star \star \star} \\
(0.000)\end{array}$ \\
\hline Female & $\begin{array}{c}-0.201 \\
(0.114)\end{array}$ & $\begin{array}{c}-0.254 \\
(0.084)\end{array}$ & $\begin{array}{c}-0.203 \\
(0.085)\end{array}$ & $\begin{array}{c}-0.222 \\
(0.089)\end{array}$ & $\begin{array}{l}-0.154^{\star \star \star} \\
(0.000)\end{array}$ & $\begin{array}{l}-0.126^{\star \star \star} \\
(0.000)\end{array}$ & $\begin{array}{c}0.146 \\
(0.539)\end{array}$ & $\begin{array}{c}-0.073 \\
(0.786)\end{array}$ \\
\hline Black & $\begin{array}{c}-0.110 \\
(0.555)\end{array}$ & - & $\begin{array}{c}-0.431 \\
(0.204)\end{array}$ & $\begin{array}{c}-0.617 \\
(0.110)\end{array}$ & $\begin{array}{c}0.038 \\
(0.689)\end{array}$ & $\begin{array}{c}-0.041 \\
(0.701)\end{array}$ & $\begin{array}{c}-1.078^{\star *} \\
(0.002)\end{array}$ & - \\
\hline Hispanic & $\begin{array}{l}0.573^{\star \star} \\
(0.001)\end{array}$ & - & $\begin{array}{c}-0.217 \\
(0.640)\end{array}$ & - & $\begin{array}{l}-0.411^{\star \star} \\
(0.002)\end{array}$ & - & $\begin{array}{c}-0.165 \\
(0.620)\end{array}$ & - \\
\hline Rural & $\begin{array}{c}-0.071 \\
(0.621)\end{array}$ & $\begin{array}{c}-0.167 \\
(0.306)\end{array}$ & $\begin{array}{l}1.302^{\star \star} \\
(0.006)\end{array}$ & - & $\begin{array}{c}0.007 \\
(0.955)\end{array}$ & - & $\begin{array}{l}0.800^{\star *} \\
(0.003)\end{array}$ & $\begin{array}{c}0.733^{\star} \\
(0.014)\end{array}$ \\
\hline Urban & $\begin{array}{c}0.242 \\
(0.089)\end{array}$ & $\begin{array}{c}0.297 \\
(0.116)\end{array}$ & $\begin{array}{c}-0.209 \\
(0.582)\end{array}$ & $\begin{array}{c}-0.386 \\
(0.374)\end{array}$ & $\begin{array}{c}0.234^{*} \\
(0.035)\end{array}$ & $\begin{array}{c}0.202 \\
(0.093)\end{array}$ & $\begin{array}{c}0.153 \\
(0.562)\end{array}$ & $\begin{array}{c}0.348 \\
(0.309)\end{array}$ \\
\hline Age & $\begin{array}{l}0.074^{\star * *} \\
(0.000)\end{array}$ & $\begin{array}{l}0.094^{\star \star *} \\
(0.000)\end{array}$ & $\begin{array}{c}0.573 \\
(0.130)\end{array}$ & $\begin{array}{c}0.793 \\
(0.090)\end{array}$ & $\begin{array}{c}0.084 \\
(0.414)\end{array}$ & $\begin{array}{c}0.075 \\
(0.590)\end{array}$ & $\begin{array}{c}0.046 \\
(0.188)\end{array}$ & $\begin{array}{c}-0.053 \\
(0.205)\end{array}$ \\
\hline Age squared & $\begin{array}{l}-0.001^{\star \star \star} \\
(0.000)\end{array}$ & $\begin{array}{c}-0.001^{\star \star} \\
(0.001)\end{array}$ & $\begin{array}{l}0.179^{\star \star \star} \\
(0.000)\end{array}$ & $\begin{array}{l}0.253^{\star \star \star} \\
(0.000)\end{array}$ & $\begin{array}{c}0.032^{*} \\
(0.028)\end{array}$ & $\begin{array}{c}0.004 \\
(0.841)\end{array}$ & $\begin{array}{c}-0.000 \\
(0.196)\end{array}$ & $\begin{array}{c}0.001 \\
(0.237)\end{array}$ \\
\hline Political knowledge & $\begin{array}{c}0.066 \\
(0.128)\end{array}$ & $\begin{array}{c}-0.005 \\
(0.922)\end{array}$ & $\begin{array}{c}-0.002^{\star \star \star} \\
(0.000)\end{array}$ & $\begin{array}{c}-0.002^{\star \star \star} \\
(0.000)\end{array}$ & $\begin{array}{r}-0.000^{*} \\
(0.029)\end{array}$ & $\begin{array}{c}-0.000 \\
(0.858)\end{array}$ & $\begin{array}{c}-0.374^{\star \star \star} \\
(0.000)\end{array}$ & $\begin{array}{c}-0.372^{\star \star \star} \\
(0.000)\end{array}$ \\
\hline
\end{tabular}


Table 3. (Continued)

\begin{tabular}{|c|c|c|c|c|c|c|c|c|}
\hline \multirow[b]{3}{*}{ Variables } & \multicolumn{2}{|c|}{ Supportive-policies index 1} & \multicolumn{2}{|c|}{ Supportive-policies index 2} & \multicolumn{2}{|c|}{ Punitive-policies index 1} & \multirow{2}{*}{\multicolumn{2}{|c|}{ Punitive-policies index 2}} \\
\hline & All & Non-White Hispanics & All & Non-White Hispanics & All & Non-White Hispanics & & \\
\hline & OLS & OLS & OLS & OLS & OLS & OLS & All & Non-White Hispanics \\
\hline Employer-sponsored & $\begin{array}{c}-0.061 \\
(0.659)\end{array}$ & $\begin{array}{r}-0.130 \\
(0.437)\end{array}$ & $\begin{array}{c}0.066 \\
(0.582)\end{array}$ & $\begin{array}{c}-0.131 \\
(0.383)\end{array}$ & $\begin{array}{r}-0.071^{*} \\
(0.018)\end{array}$ & $\begin{array}{c}-0.066 \\
(0.088)\end{array}$ & $\begin{array}{c}0.247 \\
(0.366)\end{array}$ & $\begin{array}{l}1.133^{\star \star \star} \\
(0.001)\end{array}$ \\
\hline Medicare & $\begin{array}{c}-0.106 \\
(0.543)\end{array}$ & $\begin{array}{c}-0.196 \\
(0.334)\end{array}$ & & $\begin{array}{c}-7.124^{\star \star \star} \\
(0.000)\end{array}$ & & $\begin{array}{l}1.928^{\star \star \star} \\
(0.000)\end{array}$ & $\begin{array}{c}0.237 \\
(0.467)\end{array}$ & $\begin{array}{c}0.440 \\
(0.269)\end{array}$ \\
\hline Constant & $\begin{array}{l}3.310^{\star \star \star} \\
(0.000)\end{array}$ & $\begin{array}{l}2.913^{\star \star \star} \\
(0.000)\end{array}$ & $\begin{array}{l}25.713^{\star \star \star} \\
(0.000)\end{array}$ & $\begin{array}{l}26.795^{\star \star \star} \\
(0.000)\end{array}$ & $\begin{array}{l}1.567^{\star \star \star} \\
(0.000)\end{array}$ & $\begin{array}{c}1.315^{\star} \\
(0.018)\end{array}$ & $\begin{array}{l}13.930^{\star \star \star *} \\
(0.000)\end{array}$ & $\begin{array}{l}14.103^{\star \star \star} \\
(0.000)\end{array}$ \\
\hline Education & Yes & Yes & Yes & Yes & Yes & Yes & Yes & Yes \\
\hline Income & Yes & Yes & Yes & Yes & Yes & Yes & Yes & Yes \\
\hline Observations & 2,010 & 1,329 & 1,956 & 1,300 & 2,010 & 1,329 & 1,986 & 1,314 \\
\hline $\mathrm{R}^{2}$ & 0.190 & 0.245 & 0.193 & 0.298 & 0.113 & 0.205 & 0.170 & 0.291 \\
\hline
\end{tabular}

Robust $\mathrm{p}$-values in parentheses ${ }^{\star \star \star} \mathrm{p}<0.001,{ }^{\star \star}{ }^{*} \mathrm{p}<0.01,{ }^{\star} \mathrm{p}<0.05$. 
with OUD are related to higher support for supportive policy options, the opposite holds for conservatives, those with high racial resentment and those who blame individual's personal choices for their OUD issues. Notably, there appears to be relatively broad support for these supportive policy options except for SIFS.

Conversely, we find that those with high levels of racial resentment, conservatives and those who consider addiction a personal choice are more supportive of punitive policies. The racial resentment findings are particularly noteworthy because they are so substantial. While overdose deaths among individuals of colour have steadily risen since 2000, the opioid epidemic still disproportionately impacts White Americans (Lippold et al. 2019; Drake et al. 2020). However, given the rising levels of racial resentment among Whites in recent years (Jardina 2019; Bartels 2020), and the research finding that racial resentment negatively influences government spending on drug programs (Nielsen et al. 2010), these findings do fit with the existing literature.

In many respects, our findings reinforce how negative stereotypes influence perceptions and subsequent policy preferences toward drug policy. A long history of research shows how racialised stereotypes perpetuated by the media during the crack cocaine epidemic increased support for more punitive policies (Hurwitz and Peffley 1997; Gilliam Jr. and Iyengar 2000; Dixon 2006; Wozniak 2020). While it is true that a focus on treatment-orientated policies regarding opioids has increased, racial inequities and racial stereotypes continue to influence drug policy (Kim et al. 2020). As reporting on the opioid epidemic shifts to focusing on how the opioid epidemic affects communities of colour, examining how racial attitudes influence public attitudes toward policies becomes increasingly important (Hale 2020; Khatri et al. 2021). Our findings show that racial resentment and attitudes toward opioid policies have real policy implications and could exacerbate the treatment disparities already present within the health system (Hall et al. 2015).

The findings for personal connections and personal experience are more nuanced. Those with personal connections are more likely to oppose jail time in general for those suffering from OUD problems. At the same time, they are more supportive of punitive policies against parents and pregnant women. For those who have experienced SUD, support is more subdued for all punitive policies except for arresting pregnant women. The gender findings, in particular, are intriguing. While not being the focus of this article, the findings here show that perceptions of gender, in addition to racial politics, may influence the public's support for punitive or supportive policies. Importantly, the effects are more pronounced for pregnant women than they are for parents in general. Future research should examine whether or not the different findings across genders can be replicated in other policy areas or are isolated to just OUD. Finally, our analyses of the punitive policies indices indicate consistent substantive effects only for ideology and personal choice.

The findings from our analysis of how Americans think about policies to combat the opioid crisis are remarkably policy-relevant. Some of these policies, most notably SIFS, while legal in the US due to a successful lawsuit in 2019 (Allyn and Winberg 2020), continue to face pushback from residents in areas like Philadelphia, San Francisco and New York. With opioid deaths increasing due to the COVID-19 pandemic (Centers for Disease Control and Prevention 2020), the political debate over whether to combat the opioid epidemic through public 
health and harm reduction mechanisms or punitive policies will continue to be front and centre across the country. By understanding levels of support for policies designed to combat the opioid epidemic, our article can help state and federal policymakers better understand public sentiment while designing future policies and choosing which existing policies to implement. At the very least, policymakers might be encouraged to pass and implement some of the supportive policies we described here that appear to have overwhelming support without having to worry about electoral backlash. Policy advocates and entrepreneurs may also be buoyed to renew their efforts to argue for more comprehensive policy solutions to the opioid epidemic while educating the public of the potential benefits of SIFS (Wood et al. 2007; Marshall et al. 2011).

The consistent importance of personal choice perceptions may reflect what Americans have learned about the epidemic from the media. Particularly, early on, news reports often framed opioid use not as a public health crisis but as a criminal justice issue (McGinty et al. 2016); these effects may be lingering, and it may take time to reframe the issue in the minds of Americans. The persistent influence of racial resentment, while in line with the existing literature, is also concerning. Misperceptions about minorities and African-Americans are persistent in American politics and the media (Santoro and Santoro 2018). These perceptions are resistant to change (Haeder et al. 2020) and, as a result, may discourage elected officials from supporting policy proposals that may lead to real or perceived electoral consequences for them. This, of course, bodes ill for the US ability to make headway in reigning in the epidemic.

Despite the importance of these findings, it is necessary to acknowledge several limitations of our study. First, our analysis does not account for differences across states in the severity of the opioid epidemic that could affect how individuals respond to policies designed to combat the epidemic. To the extent that respondents in particularly hard-hit states like West Virginia or Ohio respond differently than respondents in other states, our dataset cannot capture these distinctions, our analyses should thus be considered to focus on the national sentiment on these issues. Second, our analysis is limited in the number of policies explored in our study. While we do analyse public attitudes connected to a wide variety of proposed solutions to combat the opioid epidemic, the number of proposed solutions has been vast. For example, in 2018, the House of Representatives passed 58 bills designed to combat the opioid crisis during a two-week span alone (Killough and Mattingly 2018). While we focused on policies frequently mentioned in the public debate, exploring public attitudes about all possible policy options is beyond the scope of our article but expanding our work here to look at topics like attitudes towards treatment-based solutions is an important direction for future research.

Third, it is crucial to recognise that for some survey questions about the epidemic, including personal experiences, our survey explicitly mentions heroin. The distinct cultural politics surrounding heroin versus prescription opioids could affect individual responses, but we are unable to test for the influence of our wording choices here. Future research should examine the public's policy preferences toward heroin in comparison to prescription opioids. Fourth, our analysis utilised the Kinder and Sanders (1996) racial resentment scale commonly utilised in political science to measure White attitudes towards Blacks. However, the Kinder and 
Sanders measure has been recently critiqued for being too closely related to conservativism (DeSante and Smith 2020; Kam and Burge 2018). Future research should examine the robustness of our findings using alternative measures of racial resentment. Finally, our data collection occurred before the COVID-19 pandemic. There is ample evidence that the opioid epidemic might be exacerbated in the wake of the pandemic by both mentally challenging situations and reduced access to treatment options. As such further exploration of our findings here in light of the pandemic is warranted.

Ultimately, even with these limitations, our research represents a significant step forward to understand public opinion about the opioid epidemic and policies to combat it. Our analysis emphasises the importance of considering attributions of blame when studying opioid misuse and highlights the important roles of attribution of blame, political ideology, personal connections, personal experience and racial resentment in influencing public attitudes towards combatting the epidemic.

Data Availability Statement. Replication materials are available at https://doi.org/10.7910/DVN/FOHJ4D

Supplementary material. To view supplementary material for this article, please visit https://doi.org/10. 1017/S0143814X21000155

\section{References}

Addison SJ and Thorpe SJ (2004) Factors Involved in the Formation of Attitudes Towards Those Who Are Mentally Ill. Social Psychiatry and Psychiatric Epidemiology, 39(3): 228-234.

Allyn B and Winberg M (2020) "Philadelphia Nonprofit Opening Nation's 1st Supervised Injection Site Next Week.” NPR, February 26, 2020.

American Medical Association (2020) Reports of Increases in Opioid-related Overdose and Other Concerns during COVID Pandemic. Chicago, IL: American Medical Association.

Baker P, Grynbaum MM, Haberman M, Karni A and Buettner R (2019) "Frontlash: Race and the Development of Punitive Crime Policy.” New York Times, July 19, 2019.

Baker R, Brick JM, Bates NA, Battaglia M, Couper MP, Dever JA, Gile KJ and Tourangeau R (2013) Summary Report of the AAPOR Task Force on Non-Probability Sampling. Journal of Survey Statistics and Methodology, 1(2): 90-143.

Barry CL, Kennedy-Hendricks A, Gollust SE, Niederdeppe J, Bachhuber MA, Webster DW and McGinty EE (2016) Understanding Americans' Views on Opioid Pain Reliever Abuse. Addiction, 111(1): 85-93.

Barry CL, McGinty EE, Pescosolido BA and Goldman HH (2014) Stigma, Discrimination, Treatment Effectiveness, and Policy: Public Views About Drug Addiction and Mental Illness. Psychiatric Services, 65(10): 1269-1272.

Bartels LM (2020) Ethnic antagonism erodes Republicans' commitment to democracy. Proceedings of the National Academy of Sciences, 117(37): 22752-22759.

Beletsky L, Davis CS, Anderson E and Burris S (2008) The Law (and Politics) of Safe Injection Facilities in the United States. American Journal of Public Health, 98(2): 231-237.

Bobo LD and Johnson D (2004) A Taste for Punishment: Black and White Americans' Views on the Death Penalty and the War on Drugs. Du Bois Review: Social Science Research on Race, 1(1): 151-180.

Bullock HE (1999) Attributions for Poverty: A Comparison of Middle-Class and Welfare Recipient Attitudes 1. Journal of Applied Social Psychology, 29(10): 2059-2082.

Bullock HE, Williams WR and Limbert WM (2003) Predicting Support for Welfare Policies: The Impact of Attributions and Beliefs About Inequality. Journal of Poverty, 7(3): 35-56.

Callaghan TH and Olson A (2017) Unearthing the Hidden Welfare State: Race, Political Attitudes, and Unforeseen Consequences. Journal of Race, Ethnicity and Politics, 2(1): 63-87. 
Cameron AC and Trivedi PK (2010) Microeconometrics Using Stata, Revised ed College Station, TX: Stata Press.

Center for Disease Control and Prevention (2019) Drug Overdose Deaths. Atlanta, GA: Center for Disease Control and Prevention.

Centers for Disease Control and Prevention (2020) Overdose Deaths Accelerating During COVID-19. Atlanta, GA: Centers for Disease Control and Prevention.

Coppock A and McClellan OA (2019) Validating the Demographic, Political, Psychological, and Experimental Results Obtained from a New Source of Online Survey Respondents. Research \& Politics, 6(1): 1-14.

Corrigan PW, Kuwabara SA and O'Shaughnessy J (2009) The Public Stigma of Mental Illness and Drug Addiction: Findings From a Stratified Random Sample. Journal of Social Work, 9(2): 139-147.

Cozzarelli C, Tagler MJ and Wilkinson AV (2002) Do Middle-Class Students Perceive Poor Women and Poor Men Differently? Sex Roles, 47(11-12): 519-529.

DeSante CD and Smith CW (2020) Fear, Institutionalized Racism, and Empathy: The Underlying Dimensions of Whites' Racial Attitudes. PS: Political Science \& Politics, 53(4): 639-645.

Dixon TL (2006) Psychological Reactions to Crime News Portrayals of Black Criminals: Understanding the Moderating Roles of Prior News Viewing and Stereotype Endorsement. Communication Monographs, 73(2): 162-187.

Drake J, Charles C, Bourgeois JW, Daniel ES and Kwende M (2020) Exploring the impact of the opioid epidemic in Black and Hispanic communities in the United States. Drug Science, Policy and Law, 6: 2050324520940428.

Enns PK and Ramirez MD (2018) Privatizing punishment: Testing theories of public support for private prison and immigration detention facilities. Criminology, 56(3): 546-573.

Furnham A (1982) Why are thePoor Always With Us? Explanations for Poverty in Britain. British Journal of Social Psychology, 21(4): 311-322.

Gilliam Jr, FD and Iyengar S (2000) "Prime Suspects: The Influence of Local Television News on the Viewing Public." American Journal of Political Science, 44(3): 560-573.

Goodyear K, Haass-Koffler CL and Chavanne D 2018. "Opioid Use and Stigma: The Role of Gender, Language and Precipitating Events." Drug and Alcohol Dependence, 185, 339-346.

Griffin WE and Oheneba-Sakyi Y (1993) Sociodemographic and Political Correlates of University Students' Causal Attributions for Poverty. Psychological Reports, 73(3): 795-800.

Grogan CM and Park S (2017) The Politics of Medicaid: Most Americans Are Connected to the Program, Support Its Expansion, and Do Not View It as Stigmatizing. The Milbank Quarterly, 95(4): 749-782.

Grogan CM and Park S (2018) Medicaid Retrenchment Politics: Fragmented or Unified? Journal of Aging ¿ Social Policy, 30(3-4): 372-399.

Haeder SF (2020) Political Science and U.S. Health Policy in the Era of the Affordable Care Act. Policy Studies Journal, 48(S1): S14-S32.

Haeder SF, Sylvester S and Callaghan TH (2020) Lingering Legacies: Public Attitudes About Medicaid Beneficiaries and Work Requirements. Journal of Health Politics, Policy and Law, 46(2): 305-355.

Haeder SF, Sylvester S and Callaghan TH (2021) "Shared Stigma: The Effect of LGBT Status on Attitudes about the Opioid Epidemic” World Medical \& Health Policy 13 (2). https://doi.org/10.1002/wmh3.391.

Haider-Markel DP and Joslyn MR (2008) Beliefs About the Origins of Homosexuality and Subsequent Support for Gay Rights: An Empirical Test of Attribution Theory. Public Opinion Quarterly, 72, 291-310.

Haider-Markel DP and Joslyn MR (2018) Nanny State" Politics: Causal Attributions About Obesity and Support for Regulation. American Politics Research, 46(2): 199-216.

Hale K (2020) “Opioid Epidemic Weighs Financially On Black Community.” Forbes, February 20, 2020. https:// www.forbes.com/sites/korihale/2020/02/20/opioid-epidemic-weighs-financially-on-black-community/?sh= $27 \mathrm{~b} 61 \mathrm{a} 9 \mathrm{~b} 7653$.

Hall WJ, Chapman MV, Lee KM, Merino YM, Thomas TW, Payne BK, Eng E, Day SH and CoyneBeasley T (2015) Implicit racial/ethnic bias among health care professionals and its influence on health care outcomes: a systematic review. American Journal of Public Health, 105(12): e60-e76.

Harell A, Soroka S and Iyengar S (2016) Race, prejudice and attitudes toward redistribution: A comparative experimental approach. European Journal of Political Research, 55(4): 723-744. 
Harris M (2017) An Experiment Helps Heroin Users Test Their Street Drugs for Fentanyl. National Public Radio. https://www.npr.org/sections/health-shots/2017/05/16/527924429/an-experiment-helps-heroinusers-test-their-street-drugs-for-fentanyl (accessed 16 May 2017).

Heider F (1944) Social Perception and Phenomenal Causality. Psychological Review, 51(6): 358-374.

Heider F (1958) The Psychology of Interpersonal Relations. Hillsdale, NJ: Lawrence Erlbaum Associates, Inc.

Hopkins DJ (2009) Partisan Reinforcement and the Poor: The Impact of Context on Explanations For Poverty. Social Science Quarterly, 90(3): 744-764.

Hurwitz J and Peffley M (1997) Public perceptions of race and crime: The role of racial stereotypes. American Journal of Political Science, 375-401.

Jardina A (2019) White identity politics. Cambridge University Press.

Jeong S-H, Yum J and Hwang Y (2018) Effects of Media Attributions on Responsibility Judgments and Policy Opinions. Mass Communication and Society, 21(1): 24-49.

Joslyn MR and Haider-Markel DP (2017) Gun Ownership and Self-Serving Attributions for Mass Shooting Tragedies. Social Science Quarterly, 98(2): 429-442.

Kaiser Family Foundation (2016) Kaiser Health Tracking Poll: April 2016.. Menlo Park, CA: Kaiser Family Foundation.

Kam CD and Burge CD (2018) Uncovering Reactions to the Racial Resentment Scale Across the Racial Divide. The Journal of Politics, 80(1): 314-320.

Kennedy-Hendricks A, Barry CL, Gollust SE, Ensminger ME, Chisolm MS and McGinty EE (2017) Social Stigma Toward Persons With Prescription Opioid Use Disorder: Associations With Public Support for Punitive And Public Health-Oriented Policies. Psychiatric Services, 68(5): 462-469.

Khatri UG, Pizzicato LN, Viner K, Bobyock E, Sun M, Meisel ZF and South EC (2021) Racial/Ethnic Disparities in Unintentional Fatal and Nonfatal Emergency Medical Services-Attended Opioid Overdoses During the COVID-19 Pandemic in Philadelphia. JAMA Network Open, 4(1): e2034878e2034878.

Killough A and Mattingly P (2018) House approves massive opioids legislation. CNN, https://www.cnn. com/2018/06/22/politics/house-opioids-bill/index.html (accesses 22 June 2018).

Kim JW, Morgan E and Nyhan B (2020) Treatment versus Punishment: Understanding Racial Inequalities in Drug Policy. Journal of Health Politics, Policy and Law, 45(2): 177-209.

Kinder DR and Sanders LM (1996) Divided by Color: Racial Politics and Democratic Ideals. Chicago, IL: University of Chicago Press.

Krimmel K and Rader K (2017) The federal spending paradox: Economic self-interest and symbolic racism in contemporary fiscal politics. American Politics Research, 45(5): 727-754.

Levay KE, Freese J and Druckman JN (2016) The Demographic and Political Composition of Mechanical Turk Samples. Sage Open, 6(1): 2158244016636433.

Lippold KM, Jones CM, Olsen EOM and Giroir BP (2019) Racial/ethnic and age group differences in opioid and synthetic opioid-involved overdose deaths among adults aged $\geq 18$ years in metropolitan areas-United States, 2015-2017. Morbidity and Mortality Weekly Report, 68(43): 967.

Long S and Freese J (2014) Regression Models for Categorical Dependent Variables Using Stata, Third Edition. College Station, TX: Stata Press.

Lopez G (2017) “The Opioid Epidemic, Explained.” Vox, December 21, 2017. https://www.vox.com/scienceand-health/2017/8/3/16079772/opioid-epidemic-drug-overdoses.

Lopez G (2019) The Thousands of Lawsuit Against Opioid Comapnies, Explained, https://www.vox.com/ policy-and-politics/2017/6/7/15724054/opioid-epidemic-lawsuits-purdue-oxycontin（accessed 17 October 2019).

Marshall BDL, Milloy MJ, Wood E, Montaner JSG and Kerr T (2011) Reduction in overdose mortality after the opening of North America's first medically supervised safer injecting facility: a retrospective population-based study. The Lancet, 377(9775): 1429-1437.

McGinty EE, Barry CL, Stone EM, Niederdeppe J, Kennedy-Hendricks A, Linden S and Sherman SG (2018) Public support for safe consumption sites and syringe services programs to combat the opioid epidemic. Preventive Medicine, 111, 73-77.

McGinty EE, Kennedy-Hendricks A, Baller J, Niederdeppe J, Gollust S and Barry CL (2016) Criminal Activity Or Treatable Health Condition? News Media Framing of Opioid Analgesic Abuse in the United States, 1998-2012. Psychiatric Services, 67(4): 405-411. 
Morris RC and LeCount RJ (2020) The value of social control: Racial resentment, punitiveness, and white support for spending on law enforcement. Sociological Perspectives, 63(5): 697-718.

Nielsen AL, Bonn S and Wilson G (2010) Racial Prejudice and Spending on Drug Rehabilitation: The Role of Attitudes Toward Blacks And Latinos. Race and Social Problems, 2(3-4): 149-163.

Pandey J, Sinha Y, Prakash A and Tripathi RC (1982) "Right-Left Political Ideologies and Attribution of the Causes of Poverty." European Journal of Social Psychology, 12(3): 327-331.

Pew Research Center (2016) Evaluating Online Nonprobability Surveys. Washington, DC: Pew Research Center.

Quenqua D (2016) “Nancy Reagan's Most Memorable 'Just Say No’ Moments.” P.R. Week, March 7, 2016. https://www.prweek.com/article/1386405/nancy-reagans-memorable-just-say-no-moments.

Santoro TN and Santoro JD (2018) Racial Bias in the U.S. Opioid Epidemic: A Review of the History of Systemic Bias and Implications for Care. Cureus, 10(12): e3733.

Sattler S, Escande A, Racine E and Göritz AS (2017) Public Stigma Toward People With Drug Addiction: A Factorial Survey. Journal of Studies on Alcohol and Drugs, 78(3): 415-425.

Skitka LJ (1999) Ideological and Attributional Boundaries on Public Compassion: Reactions to Individuals and Communities Affected by a Natural Disaster. Personality and Social Psychology Bulletin, 25(7): 793808.

Sylvester S, Haeder SF and Callaghan TH (2021) "Replication Data for: Just Say No? Public Attitudes about Supportive and Punitive Policies to Combat the Opioid Epidemic." Harvard Dataverse: V1. https:/doi. org/10.7910/DVN/FOHJ4D

Taddonio P (2019) “Revisit Purdue Pharma's Role in the Opioid Crisis." September 12, 2019. https://www. pbs.org/wgbh/frontline/article/revisit-purdue-pharmas-role-in-the-opioid-crisis/.

Tesler M (2012) The Spillover of Racialization into Health Care: How President Obama Polarized Public Opinion by Racial Attitudes and Race. American Journal of Political Science, 56(3): 690-704.

Tesler M (2016) Post-racial or most-racial?: Race and politics in the Obama era. University of Chicago Press.

Weaver VM (2007) Frontlash: Race and the development of punitive crime policy. Studies in American Political Development, 21(2): 230-265.

Weiner B (1995) Judgments of Responsibility: A Foundation for a Theory of Social Conduct. New York: Guilford Press.

Weiner B (2006) Social Motivation, Justice, and the Moral Emotions: An Attributional Approach. New York: Psychology Press.

Wetts R and Willer R (2018) Privilege on the precipice: Perceived racial status threats lead white Americans to oppose welfare programs. Social Forces, 97(2): 793-822.

White House (2017) Remarks by President Trump Before a Briefing on the Opioid Crisis. Washington, DC: White House.

White House (2018) President Donald J. Trump's Initiative to Stop Opioid Abuse and Reduce Drug Supply and Demand. Washington, DC: White House.

Williams R (2016) Understanding and Interpreting Generalized Ordered Logit Models. The Journal of Mathematical Sociology, 40(1): 7-20.

Williams S (1984) Left-Right Ideological Differences in Blaming Victims. Political Psychology, 5(4): 573581.

Wood E, Tyndall MW, Zhang R, Montaner JSG and Kerr T (2007) Rate of detoxification service use and its impact among a cohort of supervised injecting facility users. Addiction, 102(6): 916-919.

Wootson CR Jr. (2017) "Why This Ohio Sheriff Refuses to Let His Deputies Carry Narcan to Reverse Overdoeses." Washington Post, July 8, 2017.

Wozniak KH (2020) The effect of exposure to racialized cues on white and black public support for justice reinvestment. Justice Quarterly, 37(6): 1067-1095.

Zucker GS and Weiner B (1993) Conservatism and Perceptions of Poverty: An Attributional Analysis. Journal of Applied Social Psychology, 23(12): 925-943.

Cite this article: Sylvester SM, Haeder SF, and Callaghan T (2022). Just say no? Public attitudes about supportive and punitive policies to combat the opioid epidemic. Journal of Public Policy 42, 270-297. https://doi.org/10.1017/S0143814X21000155 